\title{
IGSF3 mutation identified in patient with severe COPD alters cell function and motility
}

Kelly S. Schweitzer, ${ }^{1,2}$ Natini Jinawath, ${ }^{3,4}$ Raluca Yonescu, ${ }^{5}$ Kevin Ni, ${ }^{1,2}$ Natalia Rush, ${ }^{2}$ Varodom Charoensawan, ${ }^{4,6}$ Irina Bronova, ${ }^{1}$ Evgeny Berdyshev, ${ }^{1}$ Sonia M. Leach, ${ }^{1}$ Lucas A. Gillenwater, ${ }^{1}$ Russel P. Bowler, ${ }^{1}$ David B. Pearse, ${ }^{7}$ Constance A. Griffin, ${ }^{3}$ and Irina Petrache ${ }^{1,2,7}$

'Department of Medicine, National Jewish Health, Denver, Colorado, USA. ²Department of Medicine, Indiana University, Indianapolis, Indiana, USA. ${ }^{3}$ Program in Translational Medicine, Faculty of Medicine Ramathibodi Hospital, and ${ }^{4}$ Integrative Computational Bioscience Center, Mahidol University, Nakhon Pathom, Thailand. ${ }^{5}$ Department of Pathology, Division of Molecular Pathology, Johns Hopkins Medical Institutions, Baltimore, Maryland, USA. ${ }^{6}$ Department of Biochemistry, Faculty of Science, Mahidol University, Bangkok, Thailand. 'Department of Medicine, Johns Hopkins Medical Institutions, Baltimore, Maryland, USA.

Cigarette smoking (CS) and genetic susceptibility determine the risk for development, progression, and severity of chronic obstructive pulmonary diseases (COPD). We posited that an incidental balanced reciprocal chromosomal translocation was linked to a patient's risk of severe COPD. We determined that $46, \mathrm{XX}, \mathrm{t}(1 ; 4)(\mathrm{p} 13.1 ; \mathrm{q} 34.3)$ caused a breakpoint in the immunoglobulin superfamily member 3 (ICSF3) gene, with markedly decreased expression. Examination of COPDGene cohort identified 14 ICSF3 SNPs, of which rs1414272 and rs12066192 were directly and rs6703791 inversely associated with COPD severity, including COPD exacerbations. We confirmed that IGSF3 is a tetraspanin-interacting protein that colocalized with $C D 9$ and integrin $B 1$ in tetraspanin-enriched domains. ICSF3-deficient patient-derived lymphoblastoids exhibited multiple alterations in gene expression, especially in the unfolded protein response and ceramide pathways. IGSF3-deficient lymphoblastoids had high ceramide and sphingosine-1 phosphate but low glycosphingolipids and ganglioside levels, and they were less apoptotic and more adherent, with marked changes in multiple TNFRSF molecules. Similarly, ICSF3 knockdown increased ceramide in lung structural cells, rendering them more adherent, with impaired wound repair and weakened barrier function. These findings suggest that, by maintaining sphingolipid and membrane receptor homeostasis, ICSF3 is required for cell mobility-mediated lung injury repair. ICSF3 deficiency may increase susceptibility to CS-induced lung injury in COPD.

Authorship note: CAG is deceased.

Conflict of interest: The authors have declared that no conflict of interest exists.

Copyright: () 2020, American Society for Clinical Investigation.

Submitted: March 13, 2020

Accepted: June 10, 2020

Published: July 23, 2020.

Reference information: JCI Insight. 2020;5(14):e138101.

https://doi.org/10.1172/jici. insight.138101.

\section{Introduction}

Chronic obstructive pulmonary diseases (COPD), including emphysema, are a major cause of death worldwide. Although the most common risk factor for COPD is cigarette smoking (CS), only $30 \%$ of smokers are diagnosed with COPD, usually after the age of 50 , indicating a significant genetic component for disease susceptibility. Following the discovery that homozygosity for Glu to Lys point mutation in SERPINA1, encoding for $\alpha-1$ antitrypsin, is a genetic risk factor for COPD, there has been a keen interest in identifying other genes for COPD susceptibility. We set out to investigate if a stable balanced chromosomal translocation in a patient with manifestations of severe emphysema at a relatively young age caused a genetic disruption that increases the susceptibility of lung cells to injury.

Most stable balanced reciprocal translocations, defined by interchromosomal exchange of chromatin with no gain or loss of genetic material, do not manifest phenotypically. However, translocation breakpoints may disrupt gene regulation or expression with functional consequences. We hypothesized that the $46, \mathrm{XX}, \mathrm{t}(1 ; 4)(\mathrm{p} 13.1 ; \mathrm{q} 34.3)$ translocation present in our patient might have caused disruption of immunoglobulin superfamily member 3 (IGSF3) on chromosome 1. Little is known about the function of IGSF3 and whether it has any role in lung health, although IGSF3 SNPs have been identified in childhood asthma (1). IGSF3, by containing a Glu-Trp-Ile (EWI) motif, is subgrouped with the IGSF members EWI-2 (IGSF8), 
EWI-101 (CD101), and EWI-F (FPRP). Of these, the best characterized is EWI-2, which binds tetraspanins CD81 and CD9 (2) and links them, through the EWI motif, to the cytoskeleton to impact cell migration and proliferation. The function of IGSF3 was largely unknown until a recent report identified that it binds tetraspanin 7 (Tspan7) to control neuronal morphogenesis (3). IGSF3 is expressed in the lung (4) and in human bronchial epithelial cells (5), but its role in the lung is not known. Since IGSF3 function may be conferred via its interaction with tetraspanins, and since the double deficiency of tetraspanins CD81 and CD9 has been associated with emphysema-like phenotype in mice (6), it was conceivable that disruptions in IGSF3 may increase susceptibility to COPD or the severity of its manifestations in this patient.

In this study, we identify a loss of IGSF3 expression due to germline mutation in a patient with severe emphysema, and we define the role of IGSF3 in lung cells and show that loss of IGSF3 affects cell's sphingolipid metabolism, survival, adhesion, and wound injury repair - processes that might increase the susceptibility to CS-induced lung injury.

\section{Results}

Disruption of IGSF3 by a balanced chromosomal translocation in a patient with severe emphysema. The patient, a 45-year-old female with diffuse emphysema (representative image of her thoracic CT scan shown in Supplemental Figure 1; supplemental material available online with this article; https://doi.org/10.1172/ jci.insight.138101DS1), presented with severe lung dysfunction (forced expiratory volume at 1 second $\left[\mathrm{FEV}_{1}\right]=0.5 \mathrm{~L} / \mathrm{s} ; \mathrm{FEV}_{1} /$ forced vital capacity $[\mathrm{FVC}]=0.25$; diffusing capacity of the lungs for carbon monoxide $[\mathrm{DLCO}]=44 \%$ of predicted value and hypoxemia; arterial blood [pH 7.39], $\mathrm{pCO}_{2} 47 \mathrm{mmHg}$, $\mathrm{PaO}_{2} 72 \mathrm{mmHg}$, measured on supplemental $2 \mathrm{~L} \mathrm{O}_{2}$ ). She had a prior 15-pack-year CS history and heterozygosity for $\alpha-1$ antitrypsin deficiency (proteinase inhibitor PiMZ phenotype) with $\alpha-1$ antitrypsin levels within normal limits. When inquired about any family history of genetic abnormalities, the patient recalled that she and her son were diagnosed with a stable balanced chromosomal translocation, identified while she was pregnant. The diagnosis was prompted by an abnormal fetal ultrasound that led to amniocentesis. However, no further investigations were made because the pregnancy was uncomplicated and her son was found to be healthy. Given the relatively early onset and marked severity of this patient's emphysema relative to her CS history, we hypothesized that the germline chromosomal abnormality caused a genetic disruption that increased her susceptibility to CS-induced lung destruction.

To determine which genes were affected by the chromosomal abnormality, using an IRB-approved protocol, we collected the patient's peripheral blood lymphocytes and generated a transformed lymphoblastoid cell line. Karyotype analyses (Figure 1, A and B) confirmed the patient's history of chromosomal translocation between chromosomes 1 and 4. Using a DNA Affymetrix SNP Array 6.0 analysis, we noted that there was no genomic gain or loss in the vicinity of the breakpoint or elsewhere in the genome, consistent with a balanced translocation (data not shown). Following karyotype analyses, we mapped the chromosomal region containing the breakpoint using BAC-FISH and DNA fiber-FISH. We identified the breakpoint inside the BAC clones RP4-686J16 and RP11-763N18 on chromosome $1 \mathrm{p} 13.1$ and 4q34.3, respectively (Figure 1, C-F). Subsequent mapping, using additional BAC clones on chromosome 1p13.1, identified the breakpoint between the genomic locations 116,630,566 and 116,664,969 (GRCh38/hg38), which mapped to intron 2 of IGSF3 isoforms 1 and 2 (Figure 1G). Due to alternative splicing, these 2 isoforms differ by an additional exon ( $60 \mathrm{bp}$ ) within the IGSF3 isoform 1 (7). The counterpart of this breakpoint on chromosome 4 contained no known gene. Since the coding sequence of IGSF3 begins from exon 2, we predicted that this breakpoint may affect IGSF3 expression. Using quantitative PCR (qPCR) targeted array and immunoblotting, we found that IGSF3 mRNA and protein were greatly reduced in the patient's cells when compared with lymphoblastoid control cell lines (Figure 1, $\mathrm{H}$ and I).

IGSF3 SNPS in COPD. Since IGSF3 has not been previously associated with lung function or COPD in GWAS studies, using COPDGene data, we interrogated the frequency and impact of SNPs within or in close proximity (within $20 \mathrm{~kb}$ ) to IGSF3 gene in smokers with or without COPD, stratifying results by race. We found 8 SNPs in the intronic region; 5 SNPs in the genomic region, of which rs1414272 was within 3'UTR; and rs6703791 within exon 11 of IGSF3 (Supplemental Table 1). After adjusting for age, sex, BMI, smoking status, and pack-year smoking history, both rs1414272 — relatively common among non-Hispanic whites (NHW, 9.1\%) - and rs12066192 were significantly associated with high frequency of COPD exacerbations in NHW (Table 1). In contrast, rs6703791 (coding for a T-C missense mutation) was significantly 
A

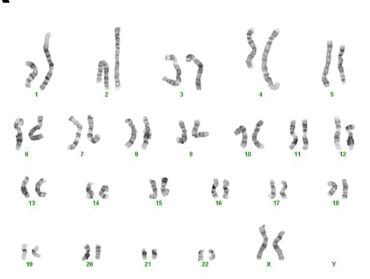

B

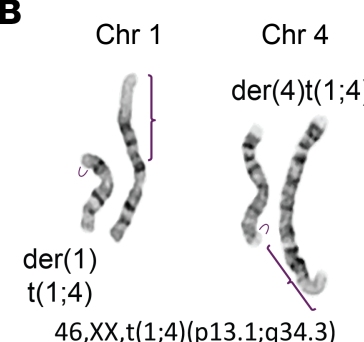

C

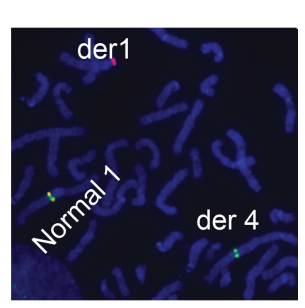

Intact

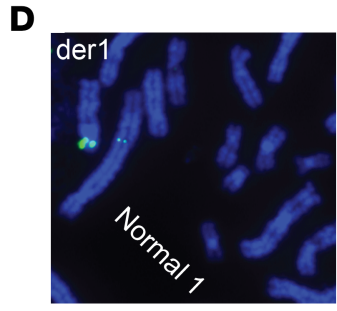

split signal RP4-686J16
E

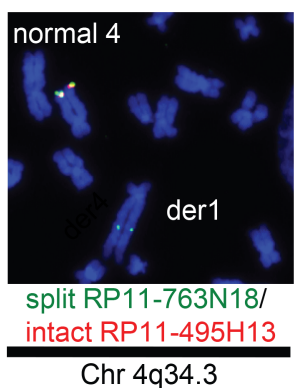

$\mathbf{F}$

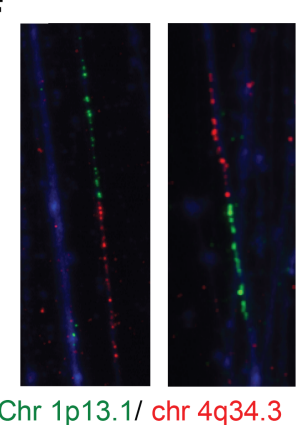

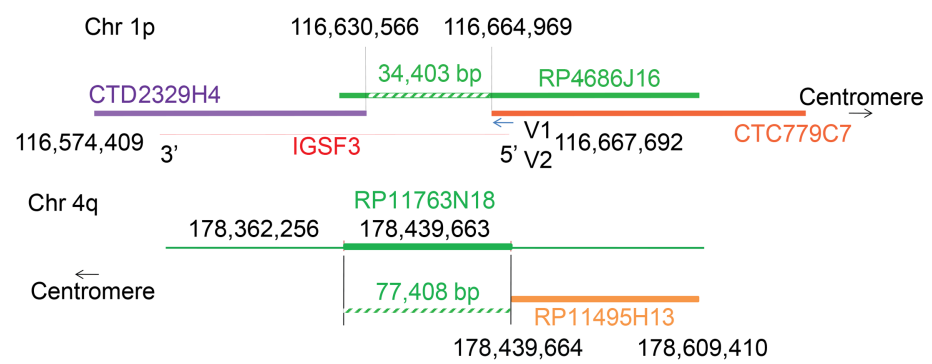

RP4-686J16/ RP11-763N18

H

Immunoglobuin superfamily member 3
I
Chr 1p13.1

G

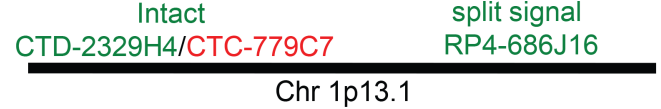


Table 1. Single nucleotide polymorphisms within 20 kb of IGSF3 locus ${ }^{A}$

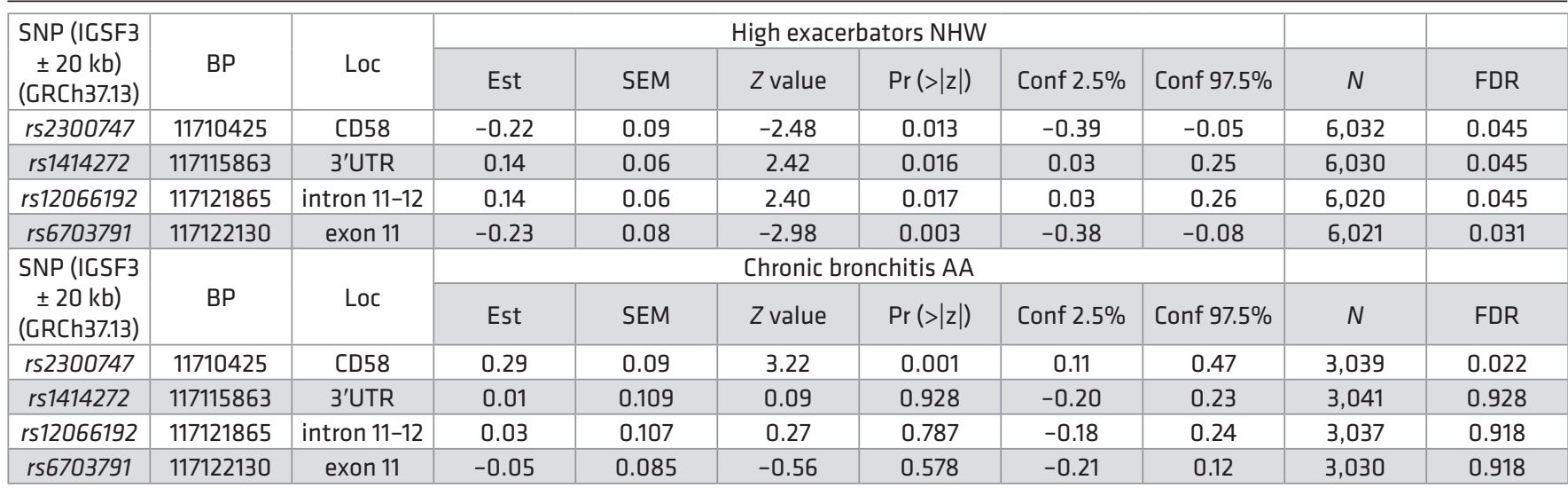

${ }^{A}$ COPDGene cohort. Loc, location; NHW, non-Hispanic whites; AA, African Americans. Est, effect estimate; Conf, Confidence interval.

IGSF3 levels in mouse lungs exposed to CS. Next, we investigated if IGSF3 levels are decreased in mouse lungs by using 2 inbred strains of mice (C57BL/ 6 and DBA2/J) that are susceptible to develop CS-induced emphysema-like phenotype. We first determined the effect of brief ( 1 day) CS exposure of C57BL/6 mice. Using qPCR, we noted that, compared with controls, lungs of CS-exposed mice had significantly decreased Igsf3 gene expression (Figure 2A). Using IHC, we next tested the effect of prolonged (4 months) CS exposures, a duration sufficient to induce emphysema-like manifestations in DBA2/J mice (8). Unlike control lungs that displayed robust and widespread IGSF3 staining in both lung parenchyma and airway epithelium, the IGSF3 immunostaining was markedly reduced in lungs of animals exposed to CS (Figure 2, B and C).

The congruent decrease in IGSF3 expression in cells from the patient with severe emphysema and in lungs of animals exposed to CS supports the notion that IGSF3 may be involved in the susceptibility or severity of lung cell responses to CS. However, since the functional significance of IGSF3 in cell biology has not been previously characterized, the mechanism by which IGSF3 would impact lung cell fate or behavior could not be inferred. We set out to determine the function of IGSF3 by comparing immortalized lymphocytes cells (lymphoblastoids) isolated from the study patient (deficient in IGSF3) with similarly transformed cells from healthy controls. We then extended these studies to cell types that are essential for the structural integrity of the lung and for gas exchange - i.e., human lung epithelial cells (using the cell line Beas2B) and primary human microvascular lung endothelial cells.

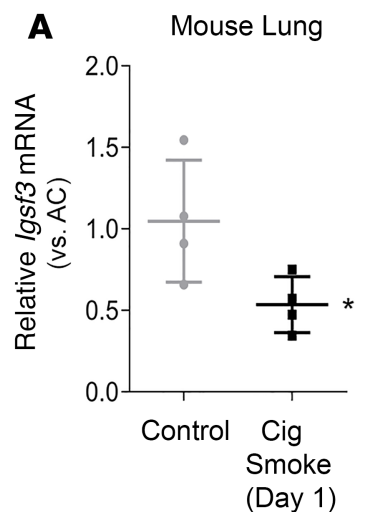

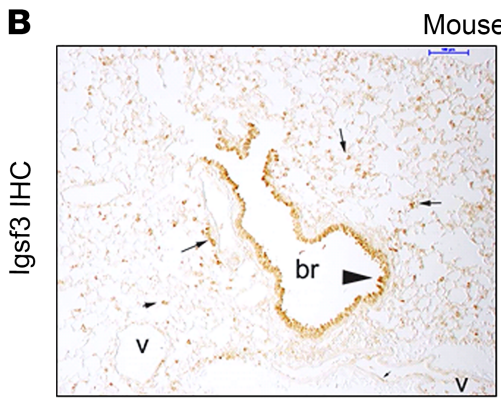

Control

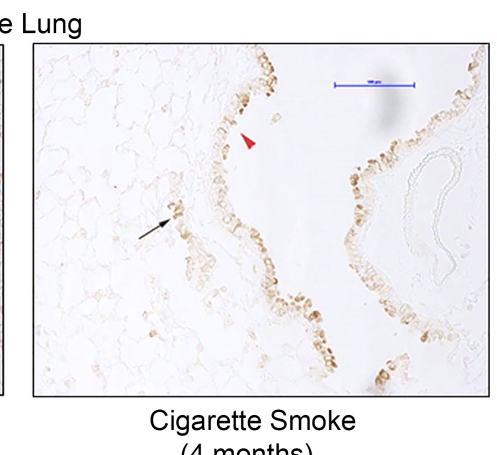

(4 months)

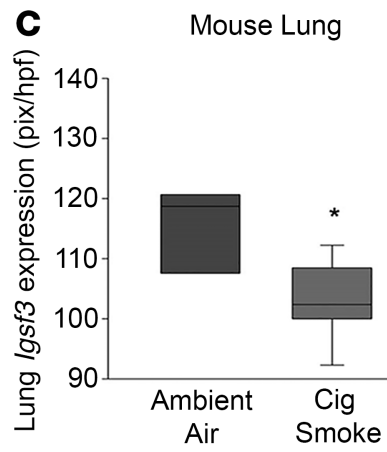

Figure 2. IGSF3 in lungs of mice exposed to cigarette smoke (CS). (A) ICSF3 mRNA in lungs of C57BL/6 mice exposed to ambient air control (AC) or CS for 1 day. ${ }^{*} P<0.05$ vs. AC, Student's $t$ test. (B) Representative images (from $n=5$ ) of IGSF3 abundance detected by IHC in lungs of DBA2/J mice exposed to AC or CS for 4 months. Scale bar: $100 \mu \mathrm{m}$. br, bronchus; v, vessel. (C) Quantification of ICSF3 abundance detected by IHC in lungs of DBA2/J mice exposed to AC or CS for 4 months. ${ }^{*} P<0.05$ vs. AC, Student's $t$ test. 
A

Tetraspanin Enriched

Microdomains (TEM)

Cell Fraction 123456789101112131415

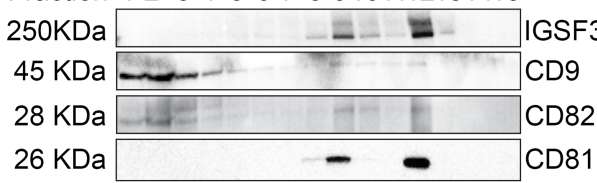

Lymphoblastoid

B

Tetraspanin Enriched Microdomains (TEM)

$\begin{array}{lllllllllllllll}\text { Cell Fraction } & 1 & 2 & 3 & 4 & 5 & 6 & 7 & 8 & 9 & 10 & 11 & 12 & 13 & 14\end{array}$

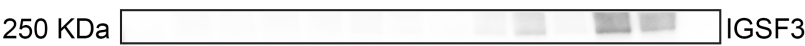

$130 \mathrm{KDa}+\mathrm{Ka}=\mathrm{m}$ ITG1 $27 \mathrm{KDa} \longrightarrow$ TSPAN7

$26 \mathrm{KDa}$

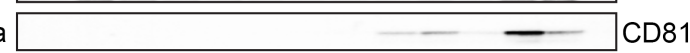

Beas2B

\section{$\mathbf{E}$}

Cell Fraction $\quad 2 \quad 3 \quad 4 \quad 5 \quad 6 \quad 7 \quad 8 \quad 910111213141516$

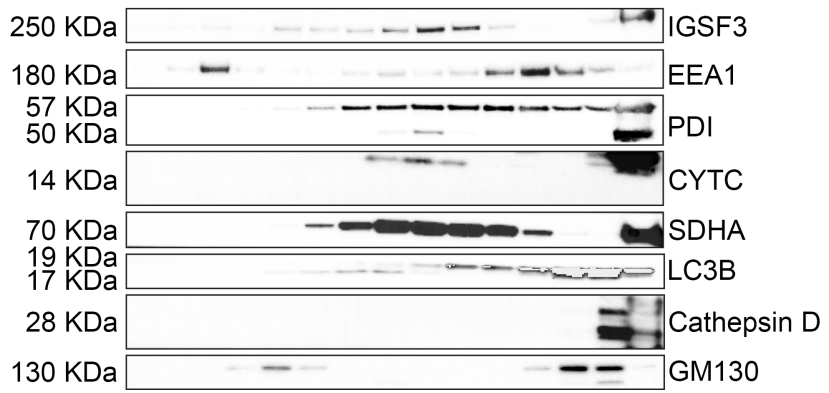

Early Endosome

Endoplasmic reticulum

Mitochondria

Lysosome

Golgi

Figure 3. ICSF3 cellular localization. (A and B) Presence of ICSF3 and indicated proteins detected by immunoblots of density gradient fractions from lymphoblastoids (A) and bronchial epithelial cells (B). (C) IGSF3 and ITCB1 detected by Western blotting in lung epithelial cells after immunoprecipitation of density gradient fractions 9 and 12 with anti-CD9 or IgG control antibodies. (D) Presence of ICSF3 or indicated proteins in either streptavidin-bound or in flow through fractions of lung epithelial cells that were cell surface biotinylated with or without CS exposure. (E) Identification of ICSF3 protein and indicated protein markers of cellular organelles in density gradient fractions of lung endothelial cells. EEA1, early endosome antigen 1; PDI, protein disulfide-isomerase; LC3B, microtubule-associated protein 1 light chain $3 \beta$; GM130, Golgi matrix protein 130 KDa; SDHA, succinate dehydrogenase complex flavoprotein subunit A; CytC, cytochrome c.

IGSF3 localization. We hypothesized that, similar to other IGSF proteins, IGSF3 interacts with tetraspanins within the plasma membrane. We performed OptiPrep density gradient ultracentrifugation to determine if IGSF3 is found within tetraspanin-enriched microdomains (TEMs). Using Western blotting of density gradient fractions of lymphoblastoid cells, we determined that tetraspanins CD82, CD9, and CD81 were all present in fractions $8-12$, and most discernably in 8,9 , and 12 , indicating that these fractions were enriched in TEMs; we noted that IGSF3 was also mostly present in these fractions (Figure 3A). Similarly, immunoblotting of density gradient fractions of Beas2B cells for tetraspanins TSPAN7 and CD81 and the tetraspanin-interacting protein integrin $\beta 1$ (ITGB1) identified fractions $8-12$, and most discernably in $8,9,11$, and 12 , as containing TEMs along with IGSF3 (Figure 3B). Using fractions 9 and 12 from Beas2B, we performed immunoprecipitation with anti-CD9 antibody followed by immunoblotting for IGSF3 and ITGB1 and established that, similar to ITGB1, IGSF3 binds tetraspanin CD9 (Figure 3C). Using fraction 9 from Beas2B cells exposed to CS extract, we noted that CS did not abolish the binding interaction between CD9 and IGSF3 (Figure 3C). These data indicate that IGSF3 coresides with tetraspanins (such as CD81, CD82, CD9, and TSPAN7) and ITGB1 in cell membranes, where it interacts with CD9.

To determine the subcellular localization of IGSF3 within membranes, we first used cell surface biotinylation, followed by IGSF3 immunoblotting of streptavidin-bound proteins. This methodology indicated the presence of IGSF3 on the cell surface, along with plasma membrane proteins CD81 and ITGB1 (Figure 3D). Exposure of cells to soluble CS extract did not alter the presence of IGSF3 at the plasma membrane (Figure 3D). We next performed IGSF3 Western blotting of density gradient fractions from rat lung endothelial cells 
Table 2. Top 30 Ingenuity Pathways significantly altered in patient lymphoblastoids ranked by abundance of upregulated genes in the respective pathway

\begin{tabular}{|c|c|c|c|c|}
\hline Ingenuity canonical pathways & Upregulated & downregulated & $-\log (P$ value $)$ & Ratio \\
\hline Unfolded protein response & $36 \%$ & $16 \%$ & 7.87 & 0.52 \\
\hline Ceramide signaling & $30 \%$ & $13 \%$ & 8.55 & 0.43 \\
\hline Acute myeloid leukemia signaling & $27 \%$ & $13 \%$ & 6.64 & 0.40 \\
\hline NGF signaling & $27 \%$ & $13 \%$ & 7.63 & 0.39 \\
\hline Telomerase signaling & $26 \%$ & $15 \%$ & 8.45 & 0.41 \\
\hline PI3K/AKT signaling & $25 \%$ & $13 \%$ & 7.29 & 0.38 \\
\hline PI3K signaling in B lymphocytes & $25 \%$ & $13 \%$ & 7.29 & 0.38 \\
\hline $\begin{array}{l}\text { Endocannabinoid developing neuron } \\
\text { pathway }\end{array}$ & $24 \%$ & $15 \%$ & 8.18 & 0.40 \\
\hline ERK/MAPK signaling & $22 \%$ & $14 \%$ & 9.17 & 0.36 \\
\hline EIF2 signaling & $21 \%$ & $21 \%$ & 15.9 & 0.41 \\
\hline Ephrin receptor signaling & $21 \%$ & $21 \%$ & 8.92 & 0.37 \\
\hline ATM signaling & $20 \%$ & $22 \%$ & 6.98 & 0.41 \\
\hline Estrogen receptor signaling & $19 \%$ & $19 \%$ & 7.41 & 0.38 \\
\hline Breast cancer regulation by Stathmin 1 & $19 \%$ & $17 \%$ & 8.83 & 0.35 \\
\hline mTOR signaling & $19 \%$ & $15 \%$ & 7.47 & 0.34 \\
\hline IL-8 signaling & $19 \%$ & $14 \%$ & 7.29 & 0.34 \\
\hline Integrin signaling & $19 \%$ & $14 \%$ & 6.24 & 0.32 \\
\hline Thrombin signaling & $19 \%$ & $13 \%$ & 6.24 & 0.32 \\
\hline CXCR4 signaling & $18 \%$ & $16 \%$ & 6.58 & 0.34 \\
\hline
\end{tabular}

(RLEC) and also probed for protein markers of cellular organelles. IGSF3 was found predominantly in density subfractions 9-11 and 16, which were shared with markers of endoplasmic reticulum (PDI), and mitochondria (CytC and SDHA), whereas IGSF3 was less prominent in fractions enriched for lysosomes (LC3B and cathep$\sin \mathrm{D}$ ), the early endosome (EEA1), or the Golgi apparatus (GM130) (Figure 3E). IGSF3 presence in fractions enriched for LC3BII (fractions 10 and 16) may suggest a potential localization in autophagosome membranes.

IGSF3 deficiency alters sphingolipid metabolism. Since tetraspanin-interacting proteins are involved in a multitude of cell functions, to better guide our interrogation of the role of IGSF3, we compared the gene expression profile of patient lymphoblastoids (deficient in IGSF3) to that of control cells from 5 healthy donors, and we input the data into Ingenuity Pathway Analysis (IPA). Of the 30 pathways that were most significantly different $(-\log [P$ values $]>6.2)$ in patient lymphoblastoid versus controls (Table 2$)$, those with the highest abundance of upregulated genes were the unfolded protein response and ceramide signaling pathways (36\% and 30\% upregulated genes, respectively). We focused on changes in the ceramide and its sphingolipid metabolic pathway, since ceramide signaling is critical for cell fate and emphysema pathogenesis (9) and since both IGSF3 and sphingolipids reside in cellular membranes. Moreover, glycosphingolipids and gangliosides are enriched in TEMs at the plasma membrane, where their clustering with tetraspanins and growth factor receptors or adhesion molecules, respectively, form plasma membrane signaling platforms dubbed glycosynapses (10). We first analyzed cDNA microarray gene expression data relative to the abundance of IGSF3 expression, given that patient cells had no IGSF3, whereas control cells from 
A

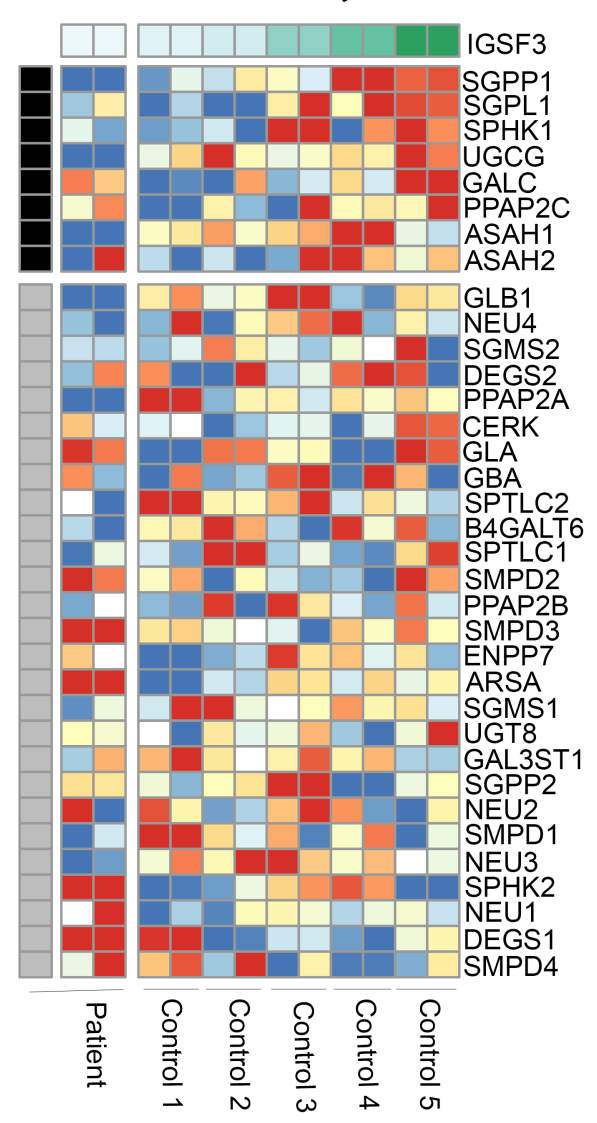

B

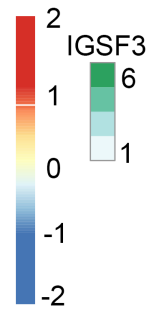

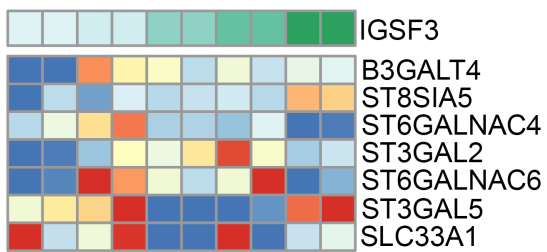

SLC33A1

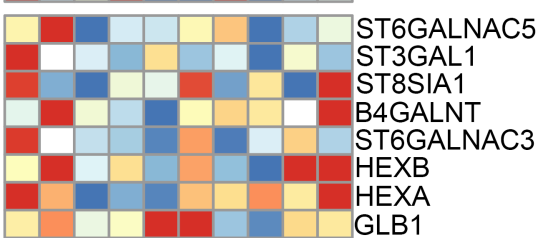

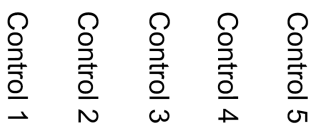

Targeted qPCR

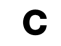

C Neutral SMase

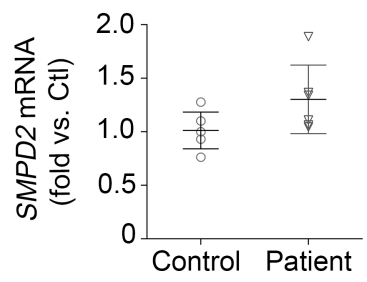

D DHC-Desaturase 1

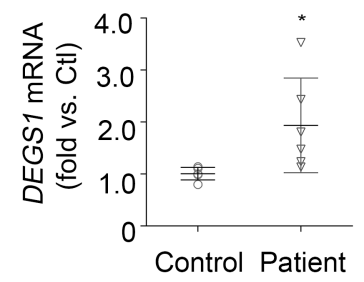

E Acid Ceramidase

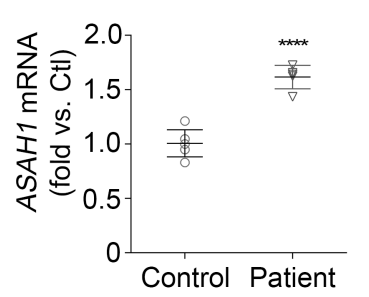

\section{$\mathbf{F}$}

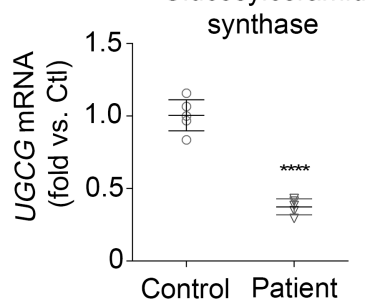

G

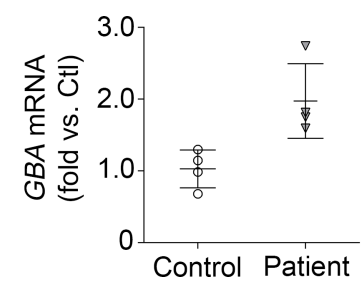

Figure 4. IGSF3 regulation of sphingolipid metabolism. (A and B) Heatmap of expression levels of genes involved in sphingolipid (A) and ganglioside (B) metabolism relative to endogenous IGSF3 expression levels, measured by CDNA microarray in lymphoblastoids from patient and 5 healthy controls. (C-G) mRNA expression levels of indicated enzymes determined by targeted qPCR. Dots represent independent experiments; mean \pm SEM shown as horizontal lines; ${ }^{*} P<0.05 ;{ }^{* * *} P<0.001$, unpaired Student's $t$ test.

5 distinct healthy donors exhibited various levels of endogenous IGSF3. IGSF3 correlated with levels of expression of multiple enzymes and transport proteins critical for the sphingolipid (Figure 4A) and ganglioside (Figure 4B) metabolism. The mRNA expression data suggest that the lower the IGSF3, the higher the expression of transcripts of enzymes that generate ceramide (Figure 4A), including all the sphingomyelinases that produce ceramide from sphingomyelin, as well as arylsulfatase A, galactosidase $\alpha$, and galactosylceramidase that produce ceramide from cerebrosides (Figure 5D, IPA). In turn, there was no evidence of increased expression of enzymes involved in de novo ceramide synthesis, with the exception of the last step of ceramide production from dihydroceramide (DHC), which showed increased expression of DHC desaturase (DEGS1). There was also increased expression of enzymes that catabolize ceramide and then generate sphingosine-1-phosphate (S1P), such as acid ceramidase (ASAH1) and sphingosine kinase 2. A notable transcriptomic signature was that of decreased expression of UGCG (glucosyl ceramide synthase) and $\beta$-1,4-galactosyltransferase 6 , the first 2 enzymes required for the synthesis of glycosylated ceramides 


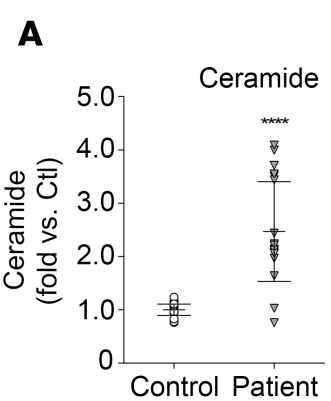

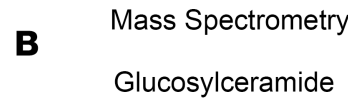

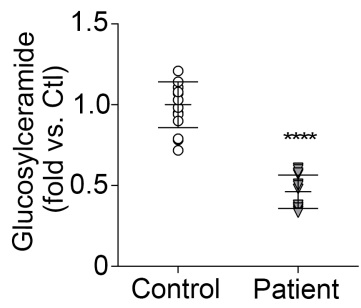

C

Trihexosylceramide

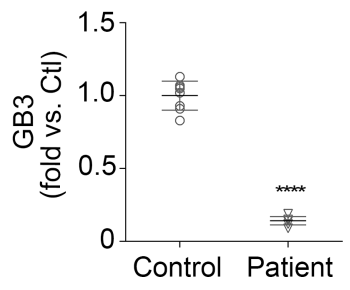

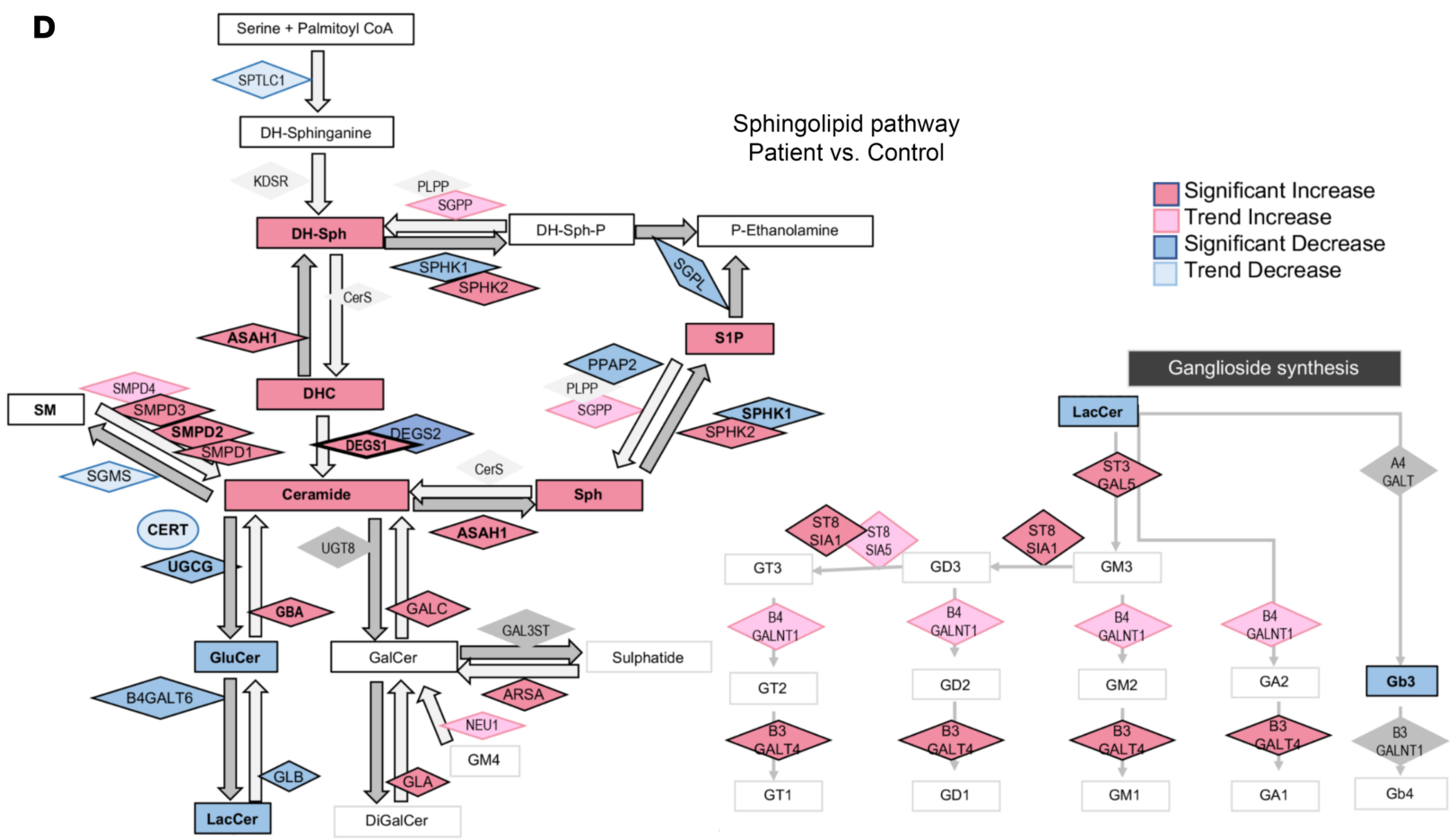

Figure 5. Role of ICSF3 on sphingolipid metabolism. (A-C) Levels of indicated metabolites measured by MS/MS. Dots represent independent experiments; mean \pm SEM shown as horizontal lines; ${ }^{* * *} P<0.001$, unpaired Student's $t$ test. (D) Schematic of sphingolipid and ganglioside metabolism with colors indicating relative changes in enzymes (mRNA) and metabolites (lipid levels) measured in patient lymphoblastoid cells compared with controls.

that eventually feed into the ganglioside synthesis pathway (Figure 5D, IPA). In turn, the transcription of enzymes that produce gangliosides in the GA, GM, GD, and GT classes was largely increased (Figure 4B and Figure 5D).

To validate the gene expression microarray data, we designed a targeted qPCR array to include select genes involved in the sphingolipid and glycosphingolipid metabolism. All results were confirmatory of the microarray findings, showing significantly increased expression of SMPD2 (neutral sphingomyelinase), $D E G S 1$, and $A S A H 1$ and significantly decreased expression of $U G C G$ in cells from patient compared with controls (Figure 4, C-G). Interestingly, we noted (data not shown) that, in the absence of IGSF3, there was a trend for decreased expression of CERT, which encodes for a ceramide transporter to Golgi, the site of complex glycosphingolipid synthesis. Since transcript levels may not be reflective on enzyme activity and output, we used tandem mass spectrometry (MS/MS) to measure select metabolites directly linked to the activity of these enzymes. Consistent with transcriptomic changes, levels of ceramide, sphingosine, and S1P were increased, and those of glucosylceramide (GluCer) and lactosylceramide (LacCer) were decreased in patient cells relative to control (Figure 5, A-C; and Table 3). Interestingly, despite increased expression of enzymes required for ganglioside synthesis, patient cells exhibited significantly decreased GB3, also known as trihexosylceramide, which was consistent with decreased production of its key precursor LacCer (Table 3). These data suggest that the loss of IGSF3 has a dramatic effect on the sphingolipid metabolic pathway, 
Table 3. Sphingolipid changes caused by decreased IGSF3 expression

\begin{tabular}{|c|c|c|c|c|c|c|c|c|c|c|}
\hline Cell type & Comparison & Ceramide & DHC & GluCer & DH-GluCer & LacCer & SM & Sph & S1P & Cer/S1P \\
\hline $\begin{array}{c}\text { Lymphoblast } \\
\qquad N=20\end{array}$ & $\begin{array}{l}\text { Patient vs. } \\
\text { control }\end{array}$ & $2.47^{*} \pm 0.21$ & $2.28^{A} \pm 0.33$ & $0.46^{A} \pm 0.03$ & $0.33^{A} \pm 0.08$ & $0.27^{A} \pm 0.03$ & $0.97 \pm 0.09$ & $1.57^{A} \pm 0.09$ & $2.07^{A} \pm 0.34$ & $1.11 \pm 0.15$ \\
\hline $\begin{array}{c}H M V E C L \\
N=13\end{array}$ & $\begin{array}{l}\text { silGSF3 vs. } \\
\text { Control }\end{array}$ & $1.46^{A} \pm 0.16$ & $1.93^{\mathrm{A}} \pm 0.24$ & $1.35^{A} \pm 0.14$ & $1.49^{A} \pm 0.12$ & $1.41^{A} \pm 0.09$ & $1.06 \pm 0.07$ & $1.46^{A} \pm 0.21$ & $0.85 \pm 0.08$ & $1.80^{A} \pm 0.19$ \\
\hline $\begin{array}{l}R L E C \\
N=8\end{array}$ & $\begin{array}{l}\text { sh/GSF3 vs. } \\
\text { Control }\end{array}$ & $1.21^{A} \pm 0.21$ & $1.21^{A} \pm 0.08$ & $3.44^{\mathrm{A}} \pm 0.37$ & $2.7^{A} \pm 0.39$ & $1.05 \pm 0.11$ & $1.01 \pm 0.03$ & $0.95 \pm 0.02$ & $0.30^{A} \pm 0.09$ & $6.28^{A} \pm 1.56$ \\
\hline
\end{tabular}

Fold changes; mean $\pm \mathrm{SD}$. ${ }^{\mathrm{A}} \mathrm{P}<0.05 . \mathrm{N}$, number of samples analyzed by tandem LQ-MS/MS; DHC, dihydroceramide; GluCer:, glucosylceramide; DH-CluCer, Dihydro-GluCer; LacCer, lactosylceramide; SM, sphingomyelin; Sph, sphingosine; S1P, sphingosine-1-phosphate; Cer/S1P, ceramide/S1P.

with impact on intracellular levels of ceramide, S1P, and LacCer, key sphingolipids with essential signaling roles required for lung tissue homeostasis.

To determine if the marked sphingolipid changes triggered by IGSF3 deficiency were cell type specific, we next analyzed the effect of IGSF3 in primary human or rat lung microvascular endothelial cells and the human bronchial epithelial cell line Beas2B using siRNA or shRNA, respectively. Similar to patient lymphoblastoids, IGSF3 knockdown increased ceramide, DHC, and sphingosine abundance (Table 3). However, unlike IGSF3-deficient lymphoblastoids, IGSF3 knockdown in either endothelial or epithelial cells markedly upregulated GluCer and decreased S1P (Table 3). This suggested that IGSF3 loss caused ceramide accumulation in all cell types tested, but its effect on GluCer and S1P was cell type specific.

Role of IGSF3 in apoptosis. Since sphingolipid metabolites such as ceramide, S1P, or GluCer determine cell fate, including survival or apoptosis, we compared patient and control lymphoblastoid cell apoptosis rates. In unstimulated, baseline culture conditions, patient lymphoblastoids had decreased active caspase-3 when compared with controls (Figure 6A). Upon UV exposure, both control and patient cells significantly activated caspase-3, but the patient cells' caspase-3 activity remained markedly lower (approximately a third) compared with controls (Figure 6A). Despite their lower baseline caspase-3 activity compared with control cells, only patient lymphoblastoids responded to CS exposure (using a soluble extract) by activating caspase-3 (Figure 6B). These results suggest that, although susceptible to environmental insults such as DNA damage or oxidative stress, lymphoblastoids lacking IGSF3 have reduced baseline apoptotic rates and may therefore have prolonged survival compared with control cells. These findings may have relevance to COPD, since lymphocytes, including B, cells are found in increased numbers in diseased lungs $(11,12)$.

To determine the molecular underpinnings of the patient lymphoblastoid phenotype, we investigated if signaling intermediates affecting cell survival were altered in the patient cells compared with controls. Western blotting demonstrated that, compared with control cells, IGSF3-deficient cells had relatively higher baseline levels of antiapoptotic phospho-AKT (Ser473) and BCL2, but with decreased antiapoptotic survivin expression and no change in phospho-FOXO1 (Figure 6C). Apoptosis-inducing CS exposure of patient cells reduced both phospho-AKT and BCL2 levels, whereas exposure of control cells to CS increased phospho-FOXO1 levels, a change associated with inhibition of apoptosis (Figure 6C). cDNA microarray data was also consistent with changes in expression of genes involved in apoptosis (Figure 6D) and suggested that many transcripts in the TNFR superfamily (TNFRSF) were significantly different in the patient cells compared with those from controls (Table 4). Although the highest magnitude of change noted was in a proapoptotic TNFRSF member, TNFRSF21 (16.3 fold) (Table 4), most changes in transcripts were consistent with an antiapoptotic response (Table 4). Next, using ELISA, we measured TNFRSF and other proteins involved in cell survival or apoptosis. We noted significantly decreased secretion of proapoptotic TNFRSF6, also known as FAS, but also of antiapoptotic TRAILR4 (decoy receptor 2 or TNFRSF10D), adiponectin (ACRP30), and receptor tyrosine kinase UFO (AXL) from patient lymphoblastoids compared with controls (Figure 6E). In turn, IGSF3-deficient (patient) cells secreted 20-fold higher levels of antiapoptotic OPG compared with control cells, independent of CS exposure (Figure 6E). These data support that IGSF3-deficient lymphocyte-derived cells' increased survival at baseline was associated with a complex predominant antiapoptotic transcriptomic and protein profile signature. Cell responses to CS exposure were characterized mostly by significant secretion of antiapoptotic AXL (TYRO7) and DTK (TYRO3) in both 
A

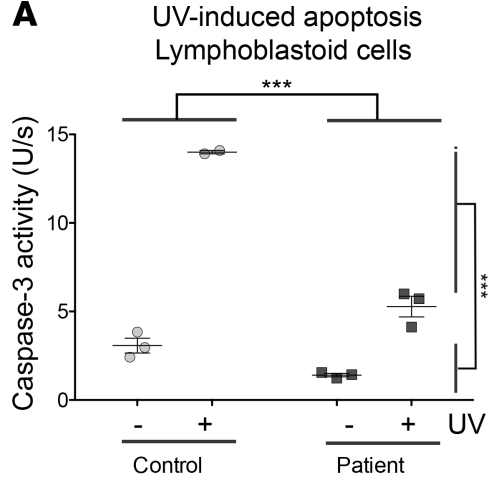

B CSE-induced apoptosis Lymphoblastoid cells

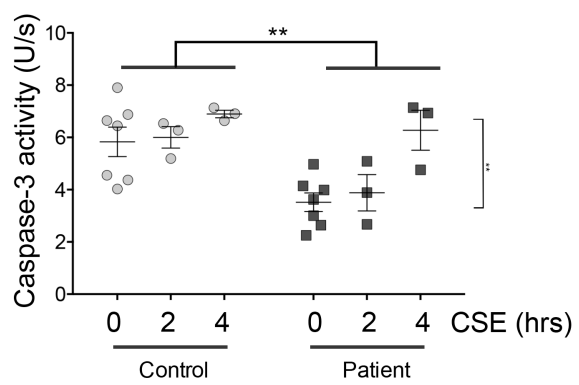

C Pro-survival signaling Lymphoblastoid cells

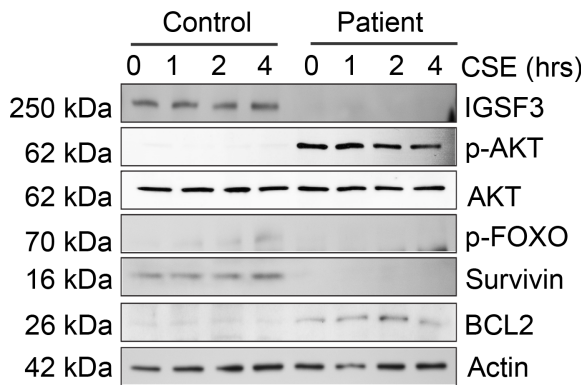

D Relative mRNA expression Apoptosis pathway Lymphoblastoid cells

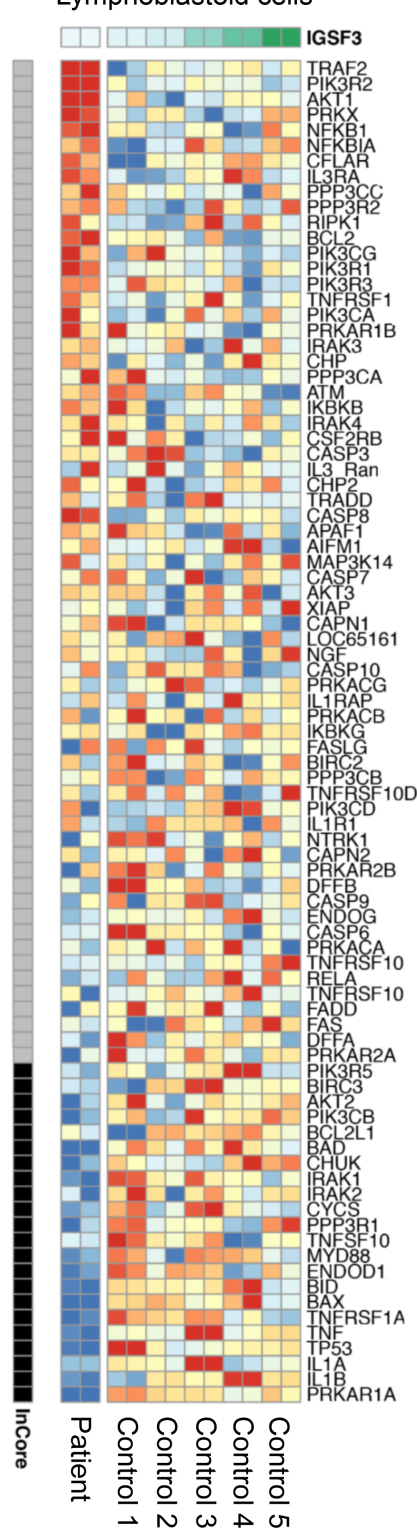

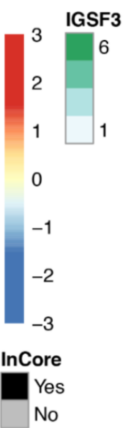

E TNFRSF and other Pro-survival Proteins Secreted Lymphoblastoid cells

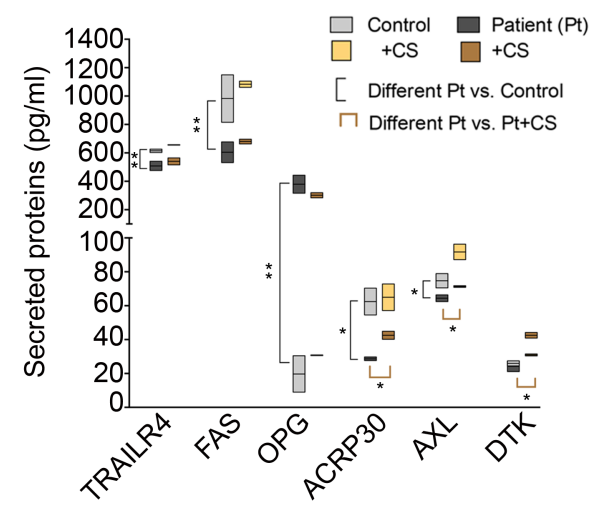

Figure 6. IGSF3 impact on apoptosis and TNFRSF expression. (A and B) Caspase-3 activity in control and patient lymphoblastoid cells exposed to ultraviolet energy (UV; A) or to CS extract (CSE); $n=3-6$ experiments, 1-way ANOVA. (C) Immunoblot of control and patient lymphoblastoids exposed to CSE (5\% v/v) for the indicated time. (D) Heatmap of mRNA expression levels of genes involved in apoptosis, relative to IGSF3 expression. Red depicts higher relative expression, and blue represents lower relative expression. (E) Secreted proteins from patient lymphoblastoids (dark gray) compared with controls (light gray) exposed to $\mathrm{CS}$; mean $\pm \mathrm{SEM} ;{ }^{*} P<0.05 ;{ }^{* *} P<0.01 ;{ }^{* *} P<0.001 ; 2$-way ANOVA.

patient and control cells and that of adiponectin in the patient's cells only (Figure 6E). Therefore, the proapoptotic effects of CS in patient cells were mostly consistent with an inhibition of AKT and BCL2 signaling.

Role of IGSF3 in cell adhesion, migration, and barrier function. In both the transcriptomic and the secreted protein profiles of patient cells compared with controls, we noted marked changes in the abundance of molecules involved in cell adhesion. For example, in patient cells, there were marked increases in the TNFRSF genes involved in cell adhesion (e.g., TNFRSF12A; 7.3-fold; Table 4), and there were decreases in ACRP30, AXL, and DTK, which are key regulators not only of cell death, but also of cell migration (Figure 6E). Indeed, microarray data reveal changes in patient cells, mostly increases, in multiple genes involved in cell adhesion (Table 5), of which the most highly upregulated were CDH6 (cadherins), claudins, and integrins. Functionally, patient cells were significantly more adherent to epithelial cells $(P<0.01)$ (Figure 7A) and 
Table 4. Changes in TNFR and TNF gene transcription in patient vs. control lymphoblastoid

\begin{tabular}{|c|c|c|c|}
\hline TNF family gene & Major function & fold change patient vs. control & $P$ value \\
\hline TNFRSF21 & proapoptotic & 16.3 & $6.2 \times 10^{-17}$ \\
\hline TNFRSF12A & adhesion & 7.3 & $1.6 \times 10^{-4}$ \\
\hline TNFRSF13B & proliferation & 3.0 & $2.6 \times 10^{-17}$ \\
\hline TNFRSF1B & antiapoptotic & 2.9 & $1.5 \times 10^{-4}$ \\
\hline TNFRSF13C & antiapoptotic & 2.5 & $3.5 \times 10^{-10}$ \\
\hline TNFSF12-TNFSF13 & proliferation & 2.5 & $1.4 \times 10^{-2}$ \\
\hline TNFRSF9 & proapoptotic & 1.5 & $3.2 \times 10^{-2}$ \\
\hline TNFRSF14 & antiapoptotic & 1.4 & $1.5 \times 10^{-3}$ \\
\hline CD40 & proapoptotic & 0.7 & $1.3 \times 10^{-4}$ \\
\hline TNFRSF1A & proapoptotic & 0.5 & $1.6 \times 10^{-2}$ \\
\hline TNF & proapoptotic & 0.4 & $4.7 \times 10^{-2}$ \\
\hline TNFRSF19 & proapoptotic & 0.3 & $1.1 \times 10^{-2}$ \\
\hline LTBR & proapoptotic & 0.030 & $2.0 \times 10^{-3}$ \\
\hline EDA2R & proapoptotic & 0.002 & $3.4 \times 10^{-2}$ \\
\hline
\end{tabular}

Relative levels (fold changes) of expression of TNFRSF genes in patient cells relative (fold changes) to average of all control cells, determined by cDNA microarray.

less migratory in a Boyden chamber assay $(P<0.01)$ compared with control cells (Figure 7B). We further determined if IGSF3 was mechanistically linked to cell adhesion by using sessile, or surface-anchored, cells such as human bronchial epithelial cells (Beas2B). Following IGSF3 knockdown via lentivirus expressing either shRNA (Figure 7C) or CRISPR-CAS9 (Figure 7E), the epithelial cell adhesion to fibronectin was markedly increased compared with control cells (Figure 7, D and F).

We next determined the functional significance of increased adhesion of surface-anchored cells by using a scratch wound closure assay in Beas2B cells. We transduced cells with lentivirus-expressing IGSF3 shRNA to knock down IGSF3 (Figure 7C), and we transfected cells with IGSF3 plasmid (pEF) to overexpress IGSF3 (Figure 8A). Loss of IGSF3 in epithelial cells decreased wound closure (Figure 8B). In turn, overexpression of IGSF3 accelerated wound repair in lung epithelial cells (Figure 8C). We next investigated IGSF3's effect on wound healing of a different surface-anchored cell type by knocking down IGSF3 in endothelial cells (Figure 8C). Primary RLEC transduced with lentivirus expressing IGSF3 shRNA (Figure $8 \mathrm{D}$ ) and primary human lung microvascular endothelial cells (HMVECL) transiently transfected with IGSF3 siRNA (Figure 8E) both had reduced wound healing compared with control cells.

Since cell adhesion and migration impact lung epithelial barrier function, which is weakened in smokers, we next assessed the role of IGSF3 in a Beas2B monolayer exposed to CS extract. Beas2B exposed to ambient air control (AC) and transfected with control shRNA or with IGSF3 shRNA maintained their barrier function for more than 12 hours (Figure $8 \mathrm{~F}$ ). Although CS exposure significantly decreased the barrier function of Beas2B transfected with either control lentivirus or IGSF3 shRNA, the latter cells had a significantly weaker barrier than control cells (Figure 8F). These data suggest that IGFS3 is required for maintenance of epithelial cell barrier function during CS exposure.

\section{Discussion}

Our studies define that IGSF3, a tetraspanin interacting protein, plays a critical role in sphingolipid homeostasis and in cell mobility. These fundamental processes in turn control cell fate and maintenance of the structural integrity of the lung, especially during injurious exposures to CS. We pursued this investigation hypothesizing that the disrupted IGSF3 transcription in our patient could have explained the severity of her emphysema. Although the patient had other risk factors for COPD and emphysema development, such as a history of moderate CS use and heterozygosity for AAT deficiency, her pulmonologists thought that neither alone or even in combination could have explained the severity of the alveolar loss at her relatively young age. We determined that the patient's chromosomal translocation 46,XX,t(1;4)(p13.1;q34.3) caused a breakpoint in the intron 2 of IGSF3 (isoforms 1 and 2) that markedly decreased the levels of this protein with unknown function.

We then analyzed COPDGene cohort data for any signal in this region that may confer a risk for COPD severity and identified several SNPs within $20 \mathrm{~kb}$ of IGSF3locus, affecting IGSF3, CD 58, and miRNA320B1. 
Table 5. Changes in adhesion molecule gene transcription in patient vs. control lymphoblastoid

\begin{tabular}{|c|c|c|}
\hline Adhesion molecule & Fold change patient vs. control & $P$ value \\
\hline CDH6 & 12.33 & $7.57 \times 10^{-2}$ \\
\hline IGSF1 & 9.88 & $3.73 \times 10^{-4}$ \\
\hline CLDN10 & 8.10 & $1.29 \times 10^{-3}$ \\
\hline CLDN16 & 7.43 & $1.86 \times 10^{-2}$ \\
\hline ITGA10 & 7.07 & $5.49 \times 10^{-3}$ \\
\hline MUCDHL & 6.61 & $2.71 \times 10^{-2}$ \\
\hline PCDHGA9 & 5.75 & $2.46 \times 10^{-2}$ \\
\hline SELL & 4.37 & $8.76 \times 10^{-6}$ \\
\hline CELSR3 & 4.30 & $1.38 \times 10^{-6}$ \\
\hline PCDHA12 & 3.67 & $1.07 \times 10^{-3}$ \\
\hline PCDH18 & 3.43 & $3.20 \times 10^{-3}$ \\
\hline CADM1 & 3.40 & $9.55 \times 10^{-3}$ \\
\hline PCDHA5 & 3.08 & $9.28 \times 10^{-3}$ \\
\hline PCDH21 & 2.79 & $4.87 \times 10^{-2}$ \\
\hline IGSF9 & 2.64 & $4.11 \times 10^{-2}$ \\
\hline CLDN23 & 2.47 & $2.73 \times 10^{-2}$ \\
\hline ITGAL & 2.29 & $1.28 \times 10^{-3}$ \\
\hline PCDHGA5 & 2.26 & $7.26 \times 10^{-2}$ \\
\hline CLDND2 & 1.97 & $8.57 \times 10^{-3}$ \\
\hline VCAM1 & 1.93 & $3.48 \times 10^{-2}$ \\
\hline ITGB7 & 1.89 & $1.08 \times 10^{-4}$ \\
\hline ITGB7 & 1.89 & $1.08 \times 10^{-4}$ \\
\hline ITGB2 & 1.42 & $5.73 \times 10^{-4}$ \\
\hline CLDN14 & 1.31 & $5.43 \times 10^{-3}$ \\
\hline ITGB4BP & 0.81 & $3.63 \times 10^{-2}$ \\
\hline ITGB1BP1 & 0.74 & $2.35 \times 10^{-2}$ \\
\hline PCDHA1 & 0.65 & $6.86 \times 10^{-2}$ \\
\hline ITGA4 & 0.59 & $4.53 \times 10^{-2}$ \\
\hline ITGB1 & 0.56 & $5.53 \times 10^{-3}$ \\
\hline CD58 & 0.52 & $3.17 \times 10^{-3}$ \\
\hline ITGAV & 0.51 & $1.18 \times 10^{-3}$ \\
\hline CELSR2 & 0.38 & $7.48 \times 10^{-2}$ \\
\hline CLDN12 & 0.33 & $3.35 \times 10^{-5}$ \\
\hline IGSF3 & 0.09 & $2.17 \times 10^{-2}$ \\
\hline CDH18 & -0.05 & $7.06 \times 10^{-3}$ \\
\hline PCDH8 & -0.45 & $2.87 \times 10^{-4}$ \\
\hline MADCAM1 & -1.03 & $4.16 \times 10^{-2}$ \\
\hline CLDN5 & -1.46 & $1.07 \times 10^{-3}$ \\
\hline IGSF2 & -2.35 & $4.98 \times 10^{-2}$ \\
\hline PCDH1 & -3.86 & $4.87 \times 10^{-2}$ \\
\hline PCDHA7 & -3.89 & $2.64 \times 10^{-3}$ \\
\hline MUPCDH & -81.76 & $8.95 \times 10^{-3}$ \\
\hline
\end{tabular}

Adhesion molecule expression identified by the cDNA microarray to be significantly different (expressed as fold change) in patient lymphoblastoids compared with control cells. Student's $t$ test was used for statistical comparisons.

After correcting for ancestry, 3 IGSF3 SNPs (rs1414272, rs12066192, and rs6703791) and 1 CD58 SNP (rs2300747) were significantly linked with COPD exacerbation frequency, a severe COPD phenotype, in NHW; the latter SNP was also significantly associated with chronic bronchitis in AA. The functional significance of these SNPs remains unknown, but rs1414272, affecting the $3^{\prime} \mathrm{UTR}$ region, might be associated with decreased IGSF3 expression. CD58 (LFA-3), a ligand for CD2, is expressed on multiple cell types, including lung epithelial cells, and is involved in Treg function and in cell adhesion (13). To our knowledge, this is the first report of IGSF3 or CD58 SNPs associated with COPD phenotypes. Previous reports indicate that IGSF3 rs 17036023 is the second-most significant SNP in asthma in European 
A Lymphoblastoid adhesion to epithelial cells

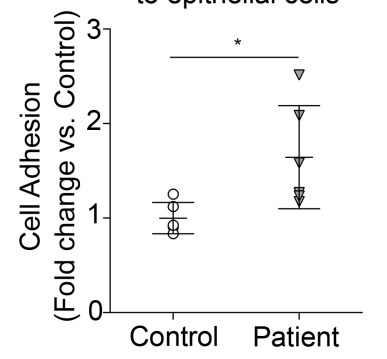

D Epithelial cell (Beas2B) adhesion to fibronectin

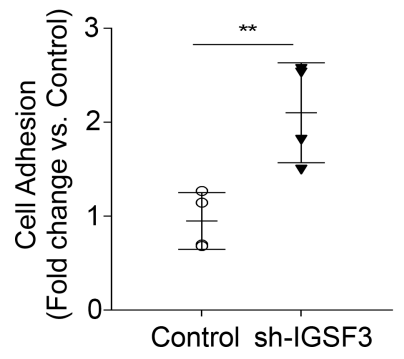

B Lymphoblastoid migration

to chemotactic stimulus

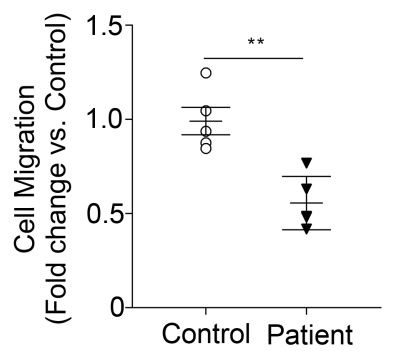

E

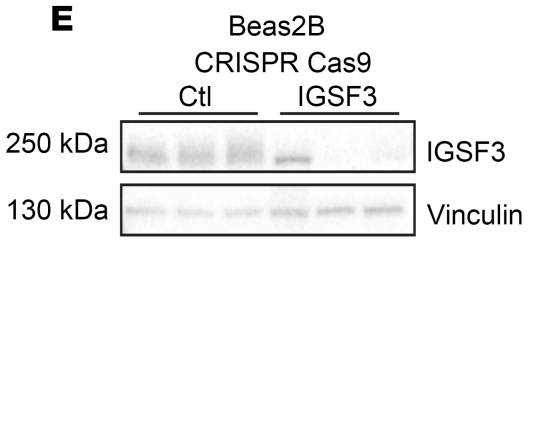

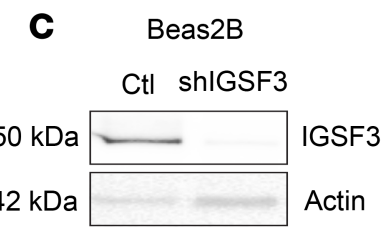

F Epithelial cell (Beas2B) adhesion to fibronectin

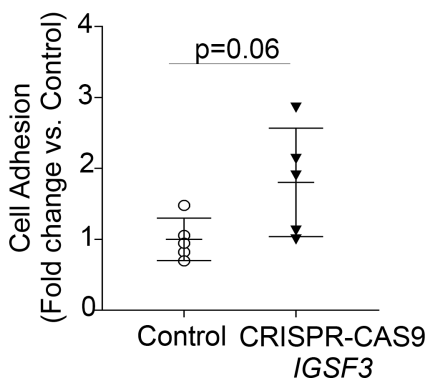

Figure 7. Role of IGSF3 in cell adhesion. (A) Cell adhesion of patient lymphoblastoids to a monolayer of cultured lung epithelial cells (Beas2B, $n=$ 3 with at least 2 replicates each, Student's $t$ test). (B) Cell migration of patient lymphoblastoids to a chemotactic stimulus (FBS) compared with controls ( $n=5$ with 3-6 replicates each, Student's $t$ test). (C) Immunoblot of IGSF3 in human bronchial epithelial cells Beas2B, stably transduced with control or IGSF3 shRNA. Actin was used as loading control. (D) Epithelial cell adhesion to fibronectin (1 hour; $n=4, P<0.001$, Student's $t$ test). (E) Immunoblot of ICSF3 in Beas2B transduced with control or ICSF3 CRISPR-CAS9. Vinculin was used as loading control. (F) Epithelial cell adhesion to fibronectin measured in Beas2B cells with CRISPR-CAS9-mediated knockdown of ICSF3 and expressed as fold change vs. control ( $n=4$ experiments, $P<0.05$, Student's $t$ test).

Americans and Hispanic Americans; rs724160030 is associated with nasolacrimal duct obstruction (14), rs647711 is associated with nasal features in Asian population (15), and CD58 SNPs are associated with autoimmune diseases and infections (16-19). In light of these reports, and our results from the COPDGene cohort (of smokers with or without COPD), our data suggest that polymorphisms in the region of IGSF3 are not associated with emphysema; instead, they may contribute to increased risk of severe airway manifestations, such as COPD exacerbations and airway infections. Our study does not rule out that other germline mutations in IGSF3 not captured in the COPDGene cohort that cause loss of IGSF3 expression confer risk for COPD or emphysema development, as in our patient.

We found that CS exposure itself can decrease IGSF3 in the airway epithelium and in the lung parenchyma, without detectable regional or cell type-specific differences in baseline IGSF3 levels or in their response to CS. Therefore, CS exposure itself may worsen the effect of any genetic loss of IGSF3 expression to further decrease the abundance of IGSF3 protein in the lung. In this study, we did not investigate the mechanisms by which CS decreases the lung expression of IGSF3 and rather focused on the functional significance of IGSF3 expression in human cell types that are relevant to CS-induced lung injury. Our results indicate that, similar to other IGSF family members, IGSF3 is anchored in TEMs enriched in tetraspanins CD81 and CD9 and integrin B1. These regions favor proteins clustering into signaling platforms that transduce external stimuli into cell responses.

Using complementary approaches of differential gene expression, bioinformatics tools for gene pathway analysis, validation of transcriptional signatures, and quantitative targeted metabolomic tools, we determined that the presence of IGSF3 is indispensable for maintaining sphingolipid and glycolipid metabolism homeostasis. We found that IGSF3 loss is promptly sensed as a stimulus to activate ceramide production. Interpretation of gene expression and metabolite abundance data implicate ceramide is produced via the sphingomyelinase pathway, but also through recycling from galactosylceramide and perhaps from sulphatides. Since ceramide is a building block for complex sphingolipids, it is conceivable that ceramide accumulation is an adaptive response to decreased GluCer and LacCer production, which are intermediate metabolites for the production of gangliosides. 
A

Beas2B

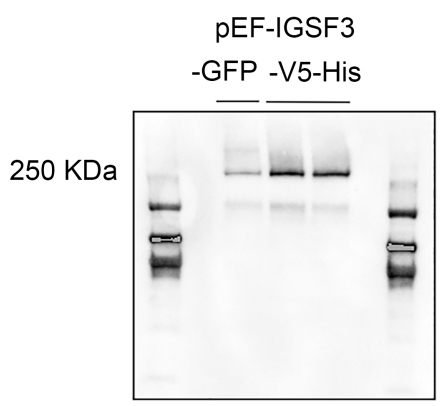
IGSF3

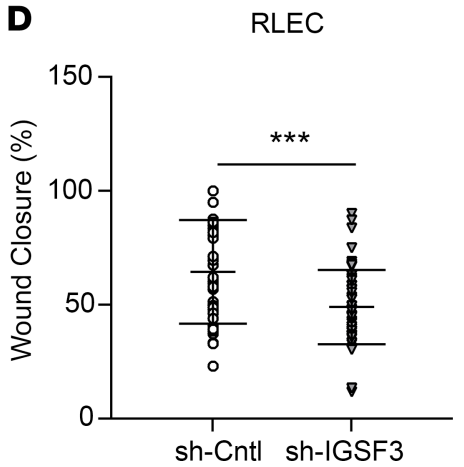

B

Beas2B

Epithelial scratch wound healing

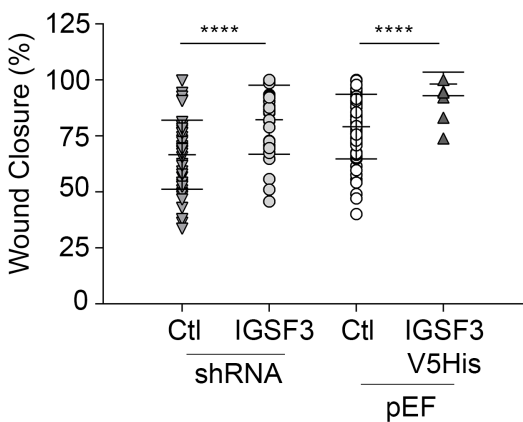

E

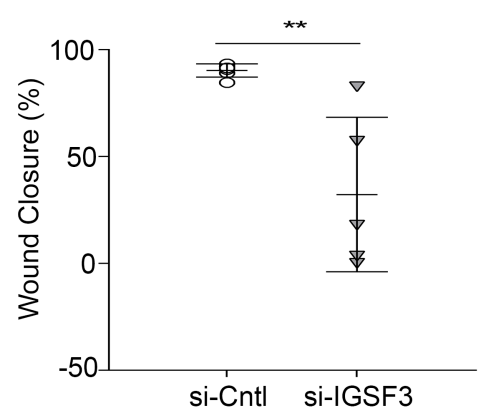

C

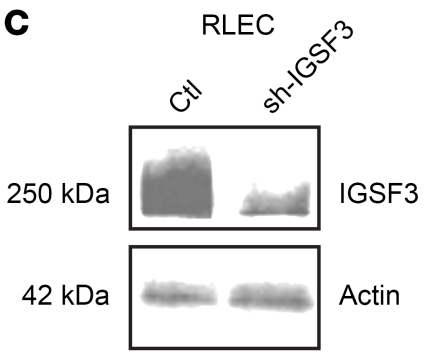

Figure 8. Role of IGSF3 in wound healing and epithelial barrier function. (A) IGSF3 immunoblot of Beas2B stably transfected with IGSF3 shRNA or pEF-IGSF3 with V5-HIS tag and respective controls. (B) Wound healing assay measured as closure (\%) of a scratch wound in Beas2B cells stably expressing shRNA to IGSF3 ( $n=9$, ${ }^{* * * *} P<0.001$, Student's $t$ test) or overexpressing pEF-IGSF3-V5His $\left(n=3\right.$, with 4 replicates, ${ }^{* * *} P<0.001$, Student's $t$ test). (C and D) IGSF3 immunoblot following knockdown in primary rat endothelial cells (RLEC) transfected with control or ICSF3 shRNA followed by closure (\%) of a scratch wound $\left(n=6,{ }^{* * *} P<0.001\right)$. (E) Wound healing in primary HMVECL transfected with IGSF3 siRNA or control siRNA $\left(n=5\right.$, ${ }^{* *} P<0.01$, Student's $t$ test). (F) Epithelial cell barrier function measured by electric cell impedance sensing (ECIS) expressed as transcellular electrical resistance (TER) normalized to the TER at the time at which CS extract was added, in confluent Beas2B cell monolayers transfected with IGSF3 shRNA or control shRNA and exposed to CS $(7.5 \%)$ or ambient air control (AC) extracts $\left(n=6-8,{ }^{*} P<0.05,{ }^{* *} P<0.01\right.$, Student's $t$ test).

A requirement for IGSF3 for ganglioside production in lymphoblastoids was supported by the marked deficiency in patient cells in the transcription of glucosyl ceramide synthase (UGCG) and B4GALT6 coupled with the deficiency in their respective products, GluCer and LacCer. Furthermore, despite upregulation of all transcripts of enzymes in the ganglioside pathway, we found low levels of GB3, the ganglioside measured in our study. The impact of IGSF3 deficiency on the glycosphingolipid metabolism was cell type specific. Transient or stable knockdown of IGSF3, in both lung endothelial and epithelial cells, led to similar increases in ceramide production and accumulation as in lymphoblast, but stimulated GluCer and LacCer production. These changes indicate that the decreased abundance of IGSF3 from TEMs, which also contain a large concentration of glycosphingolipids and gangliosides, in addition to destabilizing the structural integrity of these regions, may lead to transcriptional and enzymatic disruptions in glycosphingolipid and ganglioside pathways, an event that may be compensated differently in a cell type-specific manner. Due to yet-unclear mechanisms, in lymphoid cells, there was an overproduction of S1P at the expense of glycosphingolipids, whereas - in surface-anchored lung structural cells - the opposite was true. However, when we analyzed organelle-specific signatures of genes affected by IGSF3 loss, the most affected organelles were those that were at the same time enriched in IGSF3 and known to be involved in sphingolipid synthesis such as the ER (site of glycosphingolipid synthesis), mitochondria, and plasma membrane (Supplemental Table 2).

In patient lymphoblastoids, the increased S1P production was associated with increased resistance to UV-induced apoptosis and upregulation of activation of AKT and BLC2 signaling, along with a marked overall shift toward an increase in antiapoptotic and decrease in proapoptotic TNFRSF members. The most notable changes were the dramatic increases in antiapoptotic OPG and the downregulation of proapoptotic TRAILR4 
and FAS. The increased survival of lymphoblastoid cells deficient in IGSF3 and their increased adherence to epithelial cells may translate into increased inflammatory or lung remodeling responses at baseline or during CS. Lymphocytes, including B cells, are increased in COPD lungs (11), where they contribute to increased airway remodeling (20) and inflammation (21). Our finding of decreased mobility of patient lymphoblasts is consistent with reports that smoking may decrease migration of T lymphocytes (22) and that isolated lymphocytes from COPD blood fail to briskly migrate toward chemotactic stimuli (23). Furthermore, given the role of OPG in bone metabolism (24), and B cell development and function (25), the significance of hypersecretory OPG phenotype in IGSF3 deficiency may be of interest in COPD, which is associated with increased risk for osteoporosis and B cell proliferation. A limitation of our study is that it relied on transformed rather than primary B cells. However, key findings obtained in these patient-derived lymphoblastoids (TEM localization, increased ceramide production, and increased adherence with reduced mobility) were corroborated by similar results we noted in primary lung surface-anchored cells, endothelial, and epithelial cells.

Cells deficient in IGSF3 had markedly increased attachment to fibronectin and decreased ability to migrate to close a scratch wound, whereas cells with increased levels of IGSF3 had increased wound repair rates. The effect on cell adhesion and mobility may be related to the ability of IGSF3 to modulate cytoskeletal function through EWI and is therefore shared with other IGSF family members such as EWI2, CD101, and PTGFRN $(26,27)$. In addition, the increased adhesion could be the result of changes in receptor density due to abnormal integrin anchorage or lateral mobility induced by the marked changes in sphingolipids (28) in IGSF3-deficient cells. To our knowledge, ours is the first study of IGSFs on surface-anchored cell barrier function, showing that IGSF3 is required for lung epithelial barrier during CS exposure. This effect may be related to our finding that loss of IGSF3 weakens cell migration, which could be required for repairing breaches in the epithelial barrier during injury. Additionally, given its plasma membrane localization and link to the cytoskeleton, IGSF3 may promote intercellular tethering, a function that was not investigated in the current study. Finally, IGSF3 deficiency stimulated ceramide production, which we and others have shown to worsen the barrier function of surface-anchored lung cells $(29,30)$.

In conclusion, we have shown that the tetraspanin interacting protein IGSF3 controls sphingolipid metabolism and cell mobility, with key roles in the maintenance of lung structural and functional integrity against CS exposures. Indeed, imbalanced ceramide production at the expense of S1P or glycosphingolipids causes excessive lung endothelial cell death and emphysema-like injury (9, 31). Lung epithelial and endothelial barriers, essential in resolving inflammation, are both weakened by CS by impaired cell-to-cell adhesion and cytoskeletal rearrangement (30, 32, 33). Furthermore, the increased adherence of lymphocytes to the lung epithelium may further promote lung injury (34). Loss of IGSF3 either by genetic mutations or by CS exposure - by increasing ceramide, lymphocyte survival, and adherence and impairing wound repair and barrier function — may increase the susceptibility to CS-induced cell injury, contributing to the severity of COPD.

\section{Methods}

Reagents. All reagents were purchased from MilliporeSigma unless otherwise stated.

Chromosome analysis, BAC FISH, and DNA fiber FISH. Lymphoblastoid cells derived from B cells isolated from the patient's blood were cultured in complete RPMI 1640, and cell growth and mitotic index were used to determine harvest time. In order to determine karyotype defects, lymphoblastoids were harvested, and slides were made and G-banded using standard procedures. Twenty G-banded metaphases were analyzed, and 2 karyotypes were prepared. Chromosome abnormalities were defined and described according to the International System for Human Cytogenetic Nomenclature. FISH and DNA fiber FISH using BACs, namely CTD-2329H4 (1p13.1) labeled with SpectrumGreen, CTC-779C7 (1p13.1) labeled with SpectrumOrange, RP11-495H13 (4q34.3) labeled with SpectrumOrange, RP4-686J16 (1p13.1) labeled with SpectrumGreen, and RP11-763N18 (4q34.3) labeled with either SpectrumGreen or SpectrumOrange - obtained from the BACPAC Resource Center (Children's Hospital Oakland, Oakland, California, USA) - were performed following the manufacturer's recommended protocol and were used to further define the balanced translocation breakpoint. The signal pattern and the location of the signal were verified on 10 previously G-banded metaphases. A total of 100 interphase nuclei were also scored. For DNA fiber FISH, the DNA fiber spreading was performed using published protocols (35). The in situ hybridization steps and posthybridization washes were conducted in the same way as metaphase chromosomes, with DNA fibers counterstained with DAPI. 
Affymetrix Genome-Wide human SNP arrays 6.0 (Thermo Fisher Scientific) were used to examine genomic copy number alterations of the patient's lymphoblastoid cells. Genomic DNA was extracted using the Qiagen DNeasy Blood \& Tissue kit (catalog 69504). Array hybridization was performed according to the manufacturer's instructions and scanned in an Affymetrix GeneChip Scanner 3000. Affymetrix GeneChip Genotyping Console v3.0.1 was subsequently used for quality control, genotype calling, probe level normalization, and copy number normalization. Data analysis and visualization was performed in Chromosome analysis suite with a threshold minimum of 10 probes using NCBI build 36.

SNP analyses. Each genotyped SNP within $20 \mathrm{~kb}$ of the IGSF3 gene was tested for significant association with 5 COPD phenotypes using linear regression. The COPD phenotypes included postbronchodilator $\mathrm{FEV}_{1}$, percent predicted $\left(\mathrm{FEV}_{1} \mathrm{pp}\right), \mathrm{FEV}_{1} / \mathrm{FVC}$, chronic bronchitis (defined as self-reported chronic cough and sputum for at least 3 months in each of the 2 years prior), percent emphysema (quantified by percentage of lung voxels < -950 Hounsfield Units on the inspiratory CT scans), and high exacerbations (defined by having $>1$ events/year of acute worsening of respiratory symptoms requiring treatment with oral corticosteroids and/or antibiotics, emergency room visit, or hospital admission during long term follow-up). Models were adjusted for age, sex, BMI, smoking status, smoking pack-years, and the top 5 ancestry-based principal components, calculated as previously described (36). Associations were determined to be significant at a FDR of $5 \%$.

In vivo CS exposure. Animal studies were approved by the Animal Care and Use Committees of Indiana University. C57BL/6 or DBA2/J (females, 2 months old) were from the Jackson Laboratory. Mice were exposed to ambient air or a mixture of side stream and mainstream CS for 1 day or for 4 months (for 5 hours/day, 5 days/week), respectively, using a Teague TE-10 smoking system (Teague Enterprises) and previously published protocol (37). At the end of experiments, mice were euthanized, and the tissues were processed as previously described (8).

Routine PCR. Routine PCR was performed using GoTaq Green DNA Polymerase Master Mix (Promega, catalog M7822) using a Bio-Rad Nexus II thermal cycler. Alternatively, routine colony PCR was used to screen several colonies at once. In brief, GoTaq Green DNA Polymerase Master Mix (Promega, $25 \mu \mathrm{L}$ ) was added to PCR strips on ice. A sterile toothpick was then used to pick a single bacterial cell colony into individual PCR tubes. Colony mixtures were then amplified using standard PCR protocols and resolved on agarose gel electrophoresis.

$q P C R$. Mouse lungs were homogenized using Mini-Beadbeater-16 (Biospec). A total of $2 \mu \mathrm{g}$ of total RNA (RNeasy Mini Plus, QIAGEN) was used to reverse transcribe cDNA (High-Capacity cDNA Reverse Transcription, Thermo Fisher Scientific). qPCR was then performed on the cDNA (StepOnePlus System, Thermo Fisher Scientific) using TaqMan Universal PCR Master Mix (Thermo Fisher Scientific) and probes (all from Thermo Fisher Scientific) specific for human IGSF3 (Hs00155437_m1, lot no. 1163927), mouse IGSF3 (Mm01302155_m1, lot no. 1342279), human CERT (Hs01062552_m1, lot no. 156453), human FAPP2 (Hs01696164_s1, lot no. P161121-011 A12), human ITGB1 (Hs00559595_m1), and human GBA (Hs00164683_m1, lot no. 1611503). Relative fold expression was calculated using the $\triangle \triangle \mathrm{Ct}$ method with endogenous controls mouse GAPDH (Mm99999915_g1, lot no. 1358840) and eukaryotic 18S rRNA (Hs99999901_s1, lot no. 1505039). A targeted qPCR array (Invitrogen) was designed to measure the expression levels of human IGSF3 and genes involved in sphingolipid metabolism.

$I H C$. IHC was performed on paraffin-embedded sections using the Animal Research Kit (Agilent Dako, catalog K3954) following manufacturer specifications. In brief, sections were blocked using peroxidase. Sheep anti-human IGSF3 polyclonal antibody (1:25; R\&D Systems, catalog AF4788) was biotinylated and added to the sections and incubated overnight at $4^{\circ} \mathrm{C}$. Streptavidin was added for 15 minutes and rinsed 3 times in PBS. DAB was added for 5 minutes before mounting using Eukitt mounting medium. Microscopy was performed using a Nikon Eclipse $80 \mathrm{i}$ inverted microscope. Images were captured in a blinded fashion, and staining intensity was obtained by Metamorph Imaging software (8).

Cell culture. Lymphoblastoid control cells (transformed B cells from healthy individuals, obtained from Coriell Institute, Camden, New Jersey, USA), human bronchial airway epithelial cells (Beas2B; ATCC), and primary RLEC (obtained from Troy Stevens, University of South Alabama, Mobile, Alabama, USA) were maintained in DMEM media with high glucose supplemented with $10 \%$ FBS and 1\% penicillin/ streptomycin. Human primary microvascular endothelial cells were obtained from Lonza and maintained in their appropriate media with standard growth factor supplements (Lonza). Cells were cultured in sterile conditions at $37^{\circ} \mathrm{C}$ and $5 \% \mathrm{CO}_{2}$. 
Density gradient ultracentrifugation. Cells were grown in 4-12 T150 dishes and harvested by scraping into $0.5 \mathrm{M}$ sodium bicarbonate buffer on ice for 30 minutes. Cells were then homogenized with a dounce homogenizer and sonicated and were further incubated for 30 minutes on ice. Homogenates were then centrifuged at $20,000 \mathrm{~g}$ at $4^{\circ} \mathrm{C}$ for 10 minutes and then loaded on top of a $16 \mathrm{~mL}$ discontinuous OptiPrep (catalog D1556, lot no. MKCB1496V) gradient as follows: $5 \%, 10 \%, 20 \%$, and $40 \%$ and centrifuged at $130,000 \mathrm{~g}$ at $4^{\circ} \mathrm{C}$ for 20 hours in a Beckman Coulter Optima L-100 XP Ultracentrifuge using a SW 32.1 rotor with 17 mL buckets using Ultra-clear tubes (Beckman Coulter, catalog 344061). Fractions were collected from the top using a pipette tip into $1 \mathrm{~mL}$ fractions on ice, mixed with $4 \times$ nonreducing Laemmli buffer, and resolved by PAGE for tetraspanin localization. Another $100 \mu \mathrm{L}$ was mixed with $1 \mathrm{~mL}$ methanol for sphingolipid analyses.

Isolation of organelles. Isolation was performed as previously described (38). In brief, cells were collected by centrifugation, $500 \mathrm{~g}$ at $4^{\circ} \mathrm{C}$ for 10 minutes, and washed using HBSS. Samples were homogenized with 30 strokes in a $1 \mathrm{~mL}$ Dounce homogenizer and combined into 1 tube. Samples were then centrifuged at $20,000 \mathrm{~g}$ at $4^{\circ} \mathrm{C}$ for 10 minutes. The supernatant/cytosolic fraction was removed. The organelle fraction was then suspended in a $6 \%$ OptiPrep solution (v/v with solution " $\mathrm{C}$ " as per S53) and $500 \mu \mathrm{L}$ carbonate buffer. A $38 \mathrm{~mL}$ gradient with $3 \mathrm{~mL}$ discontinuous steps was made with v/v dilutions of OptiPrep as follows: $6 \%, 8 \%, 10 \%, 12 \%, 14 \%, 16 \%, 18 \%, 20 \%, 22 \%, 24 \%, 26 \%, 28 \%$, and $30 \%$ and centrifuged at 150,000 $g$ at $4{ }^{\circ} \mathrm{C}$ for 20 hours in a Beckman Coulter Optima XE-90 Ultracentrifuge using a SW 32.1 rotor with 38.5 $\mathrm{mL}$ buckets using Ultra-clear tubes (Beckman Coulter, catalog 344058). Fractions were collected from the bottom using a $3 \mathrm{~mL}$ syringe with a 4 -inch, 16-gauge blunt needle attached until the $2 \mathrm{~mL}$ mark on the syringe was reached. Fractions were resolved by PAGE and immunoblotted for organelle markers.

Surface biotinylation. Cells were grown on $100 \mathrm{~mm}$ culture dishes and washed $2 \times$ with cold PBS. Membrane proteins were biotinylated using Sulfo-NHS-SS-Biotin (Pierce, $5 \mathrm{mg} / \mathrm{mL}$ in PBS) for 30 minutes at $4^{\circ} \mathrm{C}$. Cells were then washed with PBS and quenched using $50 \mathrm{mM}$ Tris, pH8.0, in PBS for 5 minutes. Proteins were extracted using standard RIPA buffer with protease and phosphatase inhibitors on ice for 20 minutes. Soluble components were collected by centrifugation at $12,000 \mathrm{~g}$ at $4^{\circ} \mathrm{C}$ for 10 minutes and bound to $200 \mu \mathrm{L}$ NeutrAvidin agarose resin (Thermo Fisher Scientific, catalog 29201, lot no. QG218139) overnight. Beads were washed $3 \times$ with RIPA with protease and phosphatase inhibitors and resolved by PAGE.

Immunoblotting and immunoprecipitation. Cellular pellets were resuspended in standard RIPA buffer (catalog R0278, lot no. SLBL7395V) on ice for 30 minutes, collected by centrifugation (at $16,000 \mathrm{~g}$ at $4^{\circ} \mathrm{C}$ for 10 minutes), and quantified using standard BCA assay. Proteins were resolved by PAGE and transferred using semidry transfer apparatus (Bio-Rad) onto Immobilon-P PVDF membranes (MilliporeSigma, catalog IPVH00010). Membranes were blocked in 5\% dry milk in TBST or using Pierce Protein-Free T20 (TBS) Blocking Buffer (Thermo Fisher Scientific, catalog 37571). Antibodies were sheep anti-human IGSF3 polyclonal from R\&D Systems (catalog AF4788): CD81 (Novus, catalog NBP144861), CD9 (Novus, catalog NBP1-28363), Integrin-B1 (Novus, catalog NB100-77779), CD82 (Novus, catalog NBP1-76775), TSPAN7 (Invitrogen, catalog PA5-76938), Early endosomal marker (EEA1; catalog 3288, Cell Signaling Technology), LC3B (MilliporeSigma, catalog L7543), Cathepsin D (catalog 31718S, Cell Signaling Technology), Protein disulfide isomerase (PDI; catalog 3501, Cell Signaling Technology), GM130 (catalog 12480S, Cell Signaling Technology), Succinate dehydrogenase (SDHA; catalog 11998, Cell Signaling Technology), Cytochrome-c (CYTC; catalog 4280, Cell Signaling Technology), phospho-AKT (catalog 9271S, Cell Signaling Technology), total-AKT (catalog 9272, Cell Signaling Technology), phospho-FOXO1 (catalog 9461S, Cell Signaling Technology), survivin (R\&D Systems, catalog AF886), BCL2 (catalog 2876, Cell Signaling Technology), vinculin (Calbiochem, catalog CP74), and $\beta$-actin (MilliporeSigma, catalog A5441). Anti-mouse (catalog NA931V, lot no. 9715064) and antirabbit (catalog NA9340V, lot no. 9739636) HRP-conjugated secondary antibodies were from GE Healthcare. HRP-conjugated anti-sheep secondary antibodies were from R\&D Systems (catalog HAF106, lot no. XDP0203121). Proteins were immunoprecipitated using protein G dynabeads (Invitrogen, catalog 100.07D, lot no. 1023187) and the indicated antibody for 8 hours. Alternatively, protein G agarose beads were used. Lysates were obtained either from OptiPrep fractions, above, or from RIPA-lysed cells as indicated. Bead-antibody conjugates were incubated with indicated lysate or fraction overnight while rocking. The conjugates were then washed $4 \times$ with washing buffer or RIPA buffer and eluted with Gentle Ab-elution buffer (Invitrogen, catalog 21027). Eluates were diluted using $4 \times$ nonreducing Laemmli buffer and boiled before loading onto PAGE. Immunoprecipitations were detected using Clean Blot (Thermo Fisher Scientific, catalog 21232, lot no. PI207418) or using HRP-conjugated secondary antibodies. 
cDNA microarrays. Gene expression profiling by Illumina HT-12 beadchip array was performed using mRNAs from the $\mathrm{t}(1 ; 4)$ lymphoblastoid line $\left(I G S F 3^{+/-}\right)$and the 5 normal control lines $\left(I G S F 3^{+/+}\right)$, in duplicate (The Johns Hopkins Genetics Core). Expression values per gene in each sample were $\log _{2}$ transformed and quantile normalized using the preprocessCore package v1.32.0 (https://github.com/ bmbolstad/preprocessCore) in the R Statistical Computing Language v3.2.2 (http://www.R-project. $\mathrm{org} /$ ). A value of 100 was added to all raw signal values before being $\log _{2}$ normalized to ensure well-defined values. Genes were ranked using 1 of 2 criteria: (a) by their Pearson correlation with the IGSF3 protein levels obtained by quantitative flow cytometry data and qPCR across the 12 samples, or (b) by the fold change expression between the median patient expression and median overall control samples. Gene set enrichment analysis (GSEA) was performed on the correlation-ranked gene list using the GSEA Preranked tool within the GSEA software package, against the c2.cp.v5.2.symbols.gmt and c2.cp.kegg. v5.2.symbols.gmt gene sets within the MSigDB database (https://www.gsea-msigdb.org/gsea/msigdb/ index.jsp) (39). The default FDR of 0.25 was used to determine significantly enriched gene sets. Heatmaps of the expression profiles for genes in a given gene list were visualized using the heatmap package v1.0.8 in R, with the 'row' scaling option. Samples were annotated with their measured IGSF3 protein level, and genes were annotated as to whether or not they were core leading edge genes, as determined by GSEA. We also inputted the results into IPA (QIAGEN) for complementary assessment of pathway associations and visualization of association with the ceramide/sphingolipid pathway.

Lipid analysis. Methanol, water, and acetonitrile (HPLC grade) were purchased from Thermo Fisher Scientific. Sphingosine (Sph), dihydrosphingosine (DHSph), a 17-carbon analog of sphingosine (C17-Sph), S1P, dihydrosphingosine-1-phosphate (DHS1P), a 17-carbon analog of S1P (C17-S1P), $N$-acylated (C14:0, C16:0, C18:1, C18:0, C20:0, C24:1, C24:0) sphingosines (Ceramides, Cer), $N$-heptadecanoyl-sphingosine (C17:0-Cer), sphingomyelins $N-12: 0, N-16: 0, N-18: 0, N-24: 1$, and $N-24: 0)$, and GluCer $(N-16: 0, N-24: 1, N-$ 24:0) were from Avanti Polar Lipids. $N-\mathrm{D}_{3}-16: 0-\mathrm{GluCer}$ and $N-\mathrm{D}_{3}-16: 0-\mathrm{LacC}$ er were from Matreya LLC. The standards were dissolved in methanol (sphingoid base phosphates were dissolved with the addition of a trace amount of concentrated hydrochloric acid) and stored at $-20^{\circ} \mathrm{C}$.

Lipid extraction and sample preparation for LC-MS/MS. Lipids were extracted by modified Bligh and Dyer procedure with the use of $0.1 \mathrm{~N} \mathrm{HCl}$ for phase separation. C17-S1P (30 pmol), C17-Sph (30 pmols), $N$-C17:0-Cer (30 pmol), $N$-12:0-sphingosylphosphorylcholine (12:0-SM, 100 pmol), $N$-D -16:0-GluCer (25 pmol), and $N$ - $\mathrm{D}_{3}-16: 0$-LacCer (25 pmol) were employed as internal standards and added during the initial step of lipid extraction. Total phospholipid content was performed as described (40). Samples were concentrated under stream of nitrogen, transferred into autosampler vials, and subjected to consecutive LC-MS/ MS analysis of sphingoid bases, ceramides, glycosyl/lactosyl ceramides, and sphingoid base 1-phosphates.

Analysis of sphingoid base 1-phosphates, ceramides, sphingoid bases, sphingomyelins, and glycosyl/lactosylceramides. Analyses were performed by electrospray ionization-liquid chromatography MS/MS (ESI-LC/ MS/MS) using Sciex 6500 QTRAP hybrid triple quadrupole linear ion-trap mass spectrometer (AB Sciex) equipped with an Ion Drive Turbo V ionspray ionization source interfaced with a Shimadzu Nexera X2 UHPLC system. All lipid molecules and their derivatives were separated using Ascentis Express RP-Amide $2.7 \mu \mathrm{m} 2.1 \times 50 \mathrm{~mm}$ column. S1P and DHS1P were analyzed as bis-acetylated derivatives with C17-S1P as the internal standard employing negative ion ESI and MRM analysis as previously described. Ceramides and sphingoid bases were analyzed with C17-ceramide and C17-Sph as internal standards using positive ion ESI and MRM analysis as previously described (41). Sphingomyelin analysis was performed as previously described using methylamine reagent for 2 hours at $55^{\circ} \mathrm{C}$, evaporated with nitrogen stream, dissolved in $0.2 \mathrm{~mL}$ of methanol, and subjected to the LC-MS/MS analysis of sphingomyelins. Sphingomyelins were detected in positive ion MRM mode as a transition from corresponding molecular ions to the $\mathrm{m} / z 184$ (phosphorylcholine) and quantified by creating standard curves of variable amounts of sphingomyelin standards ( $N-16: 0-, 18: 0-, 24: 1-$, and 24:0-sphingomyelins) versus fixed amount of $N$-12:0-sphingomyelin (internal standard). Glycosylceramides and LacCer were detected in position ion MRM mode as a transition from corresponding molecular ions to the $m / z 264$. Glycosphingolipid quantitation was achieved by creating standard curves of variable amounts of GluCer standards ( $N$-16:0-, 24:1-, and 24:0-GluCer) versus a fixed amount of $N-\mathrm{D}_{3}-16: 0-\mathrm{GluCer}$ (internal standard).

Cytokine array. Human cytokine analysis was performed on supernatants collected from the patient or control lymphoblastoid cells by RayBiotech Cytokine Antibody Arrays using the Human Cytokine Antibody Array System G Series VI and VII. 
siRNA and shRNA knockdown. IGSF3 transcript was knocked down using $100 \mathrm{nM}$ siRNA (Dharmacon, siGenome siRNA, catalog M-019618-01-0005, lot nos. 090526 and 100126) for 24-72 hours using Geneporter2 (Genlantis, catalog T202007) or Lipofectamine RNAiMAX (Invitrogen, catalog 13778-150, lot nos. 1924996 and 1863033) following manufacturer's specifications. Knockdown was confirmed using qPCR or immunoblot. To create a stable cell line with knocked down IGSF3, Beas2B or RLEC were infected using 2.5 MOI of Lentiviral particles (MISSION shRNA, catalog SHCLNV-NM_001542) expressing shRNA against IGSF3 in the presence of $8 \mu \mathrm{g} / \mathrm{mL}$ polybrene (catalog H2968, lot no. SCBN6919V). Cells were infected and then, 48 hours later, were grown in full media in the presence of $5 \mu \mathrm{g} / \mathrm{mL}$ puromycin (Thermo Fisher Scientific, catalog A11138-03, lot no. 1612812) for 3 weeks. After 3 weeks, cells were split and placed into 96-wells for an additional 3-4 weeks. Resulting clones were analyzed for IGSF3 expression using qPCR and Western blotting.

IGSF3 plasmid overexpression. IGSF3 cDNA was amplified and cloned into pEF-V5-His plasmid using forward and reverse primers as follows: forward: 5'- GGACTAGTGTAGCTAGCTAGCTGCCGACATGTTCCCGGTGCTGAGCTGT-3'; reverse: 5'- ATAGTTTAGCGGCCGCTGTCTATGGCCCCTGGATGGATACTGAGAAC-3'. Phusion Hot Start II high fidelity DNA polymerase (catalog F-549S; lot no. 00384871 ) was used in a routine 2 -step reaction as follows: $98^{\circ} \mathrm{C} \times 2$ minutes, $98^{\circ} \mathrm{C}$ denaturing step for 45 seconds, $40^{\circ} \mathrm{C}$ annealing for 45 seconds, $72^{\circ} \mathrm{C}$ elongation for 4 minutes, cycled for 5 cycles; $98^{\circ} \mathrm{C} \times 45 \mathrm{sec}, 60^{\circ} \mathrm{C} \times 45 \mathrm{sec}, 72^{\circ} \mathrm{C} \times 4$ minutes for 30 cycles; $72^{\circ} \mathrm{C}$ extension step for 10 minutes. Fragments were purified using low melting point gel electrophoresis followed by QIAGEN MinElute Gel Extraction Kit (catalog 28604, lot no. 151052109). Plasmid and PCR fragments were digested using Spe1 (Thermo Fisher Scientific, catalog FD1254, lot no. 00354111) and Not1 (Thermo Fisher Scientific, catalog FD0594, lot no. 00354068) at $37^{\circ} \mathrm{C}$ overnight. The linearized plasmid and PCR fragments were gel purified as above and ligated using Roche Rapid DNA Ligation Kit (catalog 11635379001, lot no. 112498920), transformed directly into Max Efficiency DH5 $\alpha$ cells (Invitrogen, catalog 18258-012), and selected using $100 \mu \mathrm{g} / \mathrm{mL}$ carbenicillin overnight. Resulting plasmid clones were purified using Sigma Miniprep Kit (catalog PLN70, lot no. 099K6135) or QIAGEN HiSpeed Midiprep kit (catalog 12643, lot no. 151053348) and confirmed using PCR and DNA sequencing. To create a stable cell line overexpressing IGSF3, Beas2B cells were transfected with pIGSF3-V5-His or pDEST51 using Lipofectamine 2000 (Invitrogen, catalog 11668-019, lot no. 1815572), per manufacturer's specifications. After 48 hours, cell media was replaced with media supplemented with blasticidin $(4 \mu \mathrm{g} / \mathrm{mL})$.

CRISPR/CAS9-mediated knockdown of IGSF3. Knockdown of IGSF3 using CRISPR/CAS9 was achieved in Beas2B cells using GeneCopoeia lentiviral constructs. Cas9 nuclease (catalog LPP-CPLvC9NU-02-100-C) was identified under fluorescence microscopy (EGFP) and selected for using geneticin $(5 \mu \mathrm{g} / \mathrm{mL})$ for a minimum of 4 weeks. Selected colonies were then propagated and infected a second time with lentivirus containing IGSF3 or scramble gRNA (catalog LPPHCP209095L03-1-100 and catalog LPPCCPCTR01L03-100, respectively). Colonies were identified under fluorescence microscopy (mCherry) and selected for using geneticin $(5 \mu \mathrm{g} / \mathrm{mL})$ and puromycin $(0.167 \mu \mathrm{g} / \mathrm{mL})$ for at least 4 weeks. IGSF3 knockdown was confirmed using immunoblot and immunofluorescence microscopy.

Caspase-3 activity assay. Caspase-3 activity was measured with the Apo-ONE Homogenous Caspase-3/7 Assay Kit (Promega, catalog PRG7791). Human recombinant caspase-3 (VWR, catalog cc119) was used as a positive control.

Cell adhesion, cell migration/wound closure, and barrier function assays. Cell adhesion was performed using CytoSelect 96-well Cell Adhesion Assay (Cell Biolabs, catalog CBA-050, lot no. 7251316) with adhesion to fibronectin for 2 hours. Lymphocyte adhesion to epithelial cells was performed using the CytoSelect Leukocyte Epithelial Cell Adhesion Assay (Cell Biolabs, catalog CBA-211, lot no. 21320145) following manufacturer's specifications. For wound closure assays, cells were cultured in tissue culture dishes and treated as indicated. Once confluent, the cells were then scratched using a pipette tip, washed with full media, and monitored for 17 hours using a Nikon TiU microscope for images. Lymphocyte migration was performed using the CytoSelect 96-Well Cell Migration Assay ( $3 \mu \mathrm{m}$, Cell Biolabs) using FBS as a chemotactic stimulus for 4 hours, following manufacturer's recommendations. Barrier function was performed using Electric Cell-Substrate Impedance Sensing (ECIS; Applied Biophysics) using the Z-Theta 16-well array station. Cell monolayers were grown on $8 \mathrm{~W} 10 \mathrm{E}$ or $8 \mathrm{~W} 10 \mathrm{E}+$ cultureware until a capacitance of $<1 \mathrm{nF}$ or $<10 \mathrm{nF}$, respectively, at $64 \mathrm{KHz}$. Measurements of electrical resistance (Ohms) were collected at $4000 \mathrm{~Hz}$ over time.

Statistics. Analyses of significance were performed using GraphPad Prism software. A $P$ value less than 0.05 was considered statistically significant. A 2-tailed unpaired Student's $t$ test was used for 2 groups. 
One-way ANOVA was carried out when more than 2 variables were compared, and analysis-specific post hoc test were as stated in the figure legends.

Study approvals. The patient's clinical data were obtained under an IRB approved protocol (IRB no. 05-03-07-01) at The Johns Hopkins University. The blood sample from the volunteer patient was obtained via venipuncture. A portion of the blood was used for DNA extraction, to perform high-resolution chromosome analysis to confirm and identify with precision the site of the patient's balanced translocation. Part of the blood was used for establishing a stable lymphoblastoid cell line carrying the translocation. The cell line was stored in the Core Cell Center at The Johns Hopkins University using an approved institutional biosafety protocol (IBC no. B0502150109). Control human lymphoblastoids, engineered and immortalized from lymphocytes collected from patient donors, were obtained from the Coriell Institute and used under IRB-approved protocols from Indiana University (IRB nos. 0603 and 1010002369) and National Jewish Health (IRB no. HS2933). B-lymphocytes obtained from the patient were transformed into lymphoblastoids by The Johns Hopkins University Genetics Core and maintained under appropriate Institutional Review Board and Institutional Biosafety protocols at Indiana University (IRB nos. 0603-73 and 1010002369; IBC no. 1427) and National Jewish Health (IRB no. HS2993; IBC nos. BH-petrache-04-15 and BH-petrache-04-18). Animal studies were approved by the IACUC of Indiana University (IACUC no. 3027).

\section{Author contributions}

KSS contributed experimental design and conducted experiments, data analyses, and manuscript writing; NJ, RY, VC, and CAG contributed genetic analyses; KN and NR contributed experimental assistance; IB and EB contributed lipid MS; SML contributed bioinformatics; LAG and RPB contributed SNP analyses; DBP contributed patient care; and IP contributed experimental design, data analysis and interpretation, and manuscript writing.

\section{Acknowledgments}

This work was supported in part by grants sponsored by the Department of Medicine Research Microgrant, National Jewish Health (MEDRESEARCH to KS); Wollowick Chair of Pulmonary Research, National Jewish Health (IP); Calvin H. English Chair of Medical Research and Floyd and Reba Smith Chair, Indiana University (IP); award no. RHL077328 (IP); and The Crown Property Bureau Foundation Funds (NJ and VC). The SNP study using the COPDGene cohort described was supported by award nos. U01 HL089897 and U01 HL089856 from the National Heart, Lung, and Blood Institute. The content is solely the responsibility of the authors and does not necessarily represent the official views of the National Heart, Lung, and Blood Institute or the NIH. The COPDGene project is also supported by the COPD Foundation through contributions made to an Industry Advisory Board composed of AstraZeneca, Boehringer-Ingelheim, Genentech, GlaxoSmithKline, Novartis, Pfizer Siemens, and Sunovion. We thank Matthew Justice for assistance with ultracentrifugation and OptiPrep discontinuous gradients. We thank the COPDGene Biomarker Core faculty Katerina Kechris and Farnoush Banaei-Kashani for their assistance. We also thank Kathleen Murphy for her help with characterization of the patient translocation.

Address correspondence to: Irina Petrache, National Jewish Health, 1400 Jackson Street, Denver, Colorado 80206, USA. Phone: 303.270.1655; Email: petrachei@NJHealth.org.

1. Ding L, et al. Rank-based genome-wide analysis reveals the association of ryanodine receptor-2 gene variants with childhood asthma among human populations. Hum Genomics. 2013;7:16.

2. Stipp CS, Orlicky D, Hemler ME. FPRP, a major, highly stoichiometric, highly specific CD81- and CD9-associated protein. J Biol Chem. 2001;276(7):4853-4862.

3. Usardi A, Iyer K, Sigoillot SM, Dusonchet A, Selimi F. The immunoglobulin-like superfamily member IGSF3 is a developmentally regulated protein that controls neuronal morphogenesis. Dev Neurobiol. 2017;77(1):75-92.

4. Saupe S, Roizès G, Peter M, Boyle S, Gardiner K, De Sario A. Molecular cloning of a human cDNA IGSF3 encoding an immunoglobulin-like membrane protein: expression and mapping to chromosome band 1p13. Genomics. 1998;52(3):305-311.

5. So MY, et al. Gene expression profile and toxic effects in human bronchial epithelial cells exposed to zearalenone. PLoS One. 2014;9(5):e96404

6. Takeda Y, et al. Double deficiency of tetraspanins CD9 and CD81 alters cell motility and protease production of macrophages and causes chronic obstructive pulmonary disease-like phenotype in mice. J Biol Chem. 2008;283(38):26089-26097.

7. Hunt SE, et al. Ensembl variation resources. Database (Oxford). 2018;2018.

8. Clauss $\mathrm{M}$, et al. Lung endothelial monocyte-activating protein 2 is a mediator of cigarette smoke-induced emphysema in mice. 
J Clin Invest. 2011;121(6):2470-2479.

9. Petrache I, et al. Ceramide upregulation causes pulmonary cell apoptosis and emphysema-like disease in mice. $\mathrm{Nat} \mathrm{Med}$. 2005;11(5):491-498

10. Toledo MS, Suzuki E, Handa K, Hakomori S. Cell growth regulation through GM3-enriched microdomain (glycosynapse) in human lung embryonal fibroblast WI38 and its oncogenic transformant VA13. J Biol Chem. 2004;279(33):34655-34664.

11. Gosman MM, et al. Increased number of B-cells in bronchial biopsies in COPD. Eur Respir J. 2006;27(1):60-64

12. Hogg JC, et al. The nature of small-airway obstruction in chronic obstructive pulmonary disease. $N$ Engl J Med. 2004;350(26):2645-2653.

13. Bloemen PG, et al. Expression and modulation of adhesion molecules on human bronchial epithelial cells. Am J Respir Cell Mol Biol. 1993;9(6):586-593.

14. Foster J, et al. Identification of an IGSF3 mutation in a family with congenital nasolacrimal duct obstruction. Clin Genet. 2014;86(6):589-591.

15. Wu W, et al. Whole-exome sequencing identified four loci influencing craniofacial morphology in northern Han Chinese. Hum Genet. 2019;138(6):601-611.

16. Yamamoto M, et al. Association of CD58 Polymorphisms and its Protein Expression with the Development and Prognosis of Autoimmune Thyroid Diseases. Immunol Invest. 2020;49(1-2):106-119.

17. Hecker $\mathrm{M}$, et al. A genetic variant associated with multiple sclerosis inversely affects the expression of CD58 and microRNA-548ac from the same gene. PLoS Genet. 2019;15(2):e1007961.

18. Kumar V, et al. Immunochip SNP array identifies novel genetic variants conferring susceptibility to candidaemia. Nat Commun. 2014;5:4675.

19. Coustet B, et al. Association study of ITGAM, ITGAX, and CD58 autoimmune risk loci in systemic sclerosis: results from 2 large European Caucasian cohorts. J Rheumatol. 2011;38(6):1033-1038.

20. Donovan C, et al. Roles for T/B lymphocytes and ILC2s in experimental chronic obstructive pulmonary disease. J Leukoc Biol. 2019;105(1):143-150.

21. Polverino F, Seys LJ, Bracke KR, Owen CA. B cells in chronic obstructive pulmonary disease: moving to center stage. Am J Physiol Lung Cell Mol Physiol. 2016;311(4):L687-L695.

22. Koch A, et al. Modification of surface antigens in blood CD8+ T-lymphocytes in COPD: effects of smoking. Eur Respir J. 2007;29(1):42-50

23. Costa C, et al. Enhanced monocyte migration to CXCR3 and CCR5 chemokines in COPD. Eur Respir J. 2016;47(4):1093-1102.

24. Kong YY, et al. Activated T cells regulate bone loss and joint destruction in adjuvant arthritis through osteoprotegerin ligand. Nature. 1999;402(6759):304-309.

25. Yun TJ, et al. Osteoprotegerin, a crucial regulator of bone metabolism, also regulates B cell development and function. J Immunol. 2001;166(3):1482-1491.

26. He B, Zhang YH, Richardson MM, Zhang JS, Rubinstein E, Zhang XA. Differential functions of phospholipid binding and palmitoylation of tumour suppressor EWI2/PGRL. Biochem J. 2011;437(3):399-411.

27. Clark KL, Zeng Z, Langford AL, Bowen SM, Todd SC. PGRL is a major CD81-associated protein on lymphocytes and distinguishes a new family of cell surface proteins. J Immunol. 2001;167(9):5115-5121.

28. Eich C, et al. Changes in membrane sphingolipid composition modulate dynamics and adhesion of integrin nanoclusters. Sci Rep. 2016;6:20693.

29. McVey MJ, et al. Acid sphingomyelinase mediates murine acute lung injury following transfusion of aged platelets. Am J Physiol Lung Cell Mol Physiol. 2017;312(5):L625-L637.

30. Schweitzer KS, et al. Mechanisms of lung endothelial barrier disruption induced by cigarette smoke: role of oxidative stress and ceramides. Am J Physiol Lung Cell Mol Physiol. 2011;301(6):L836-L846.

31. Koike K, et al. Role of Glucosylceramide in Lung Endothelial Cell Fate and Emphysema. Am J Respir Crit Care Med. 2019;200(9):1113-1125.

32. Nishida K, et al. Cigarette smoke disrupts monolayer integrity by altering epithelial cell-cell adhesion and cortical tension. $A m J$ Physiol Lung Cell Mol Physiol. 2017;313(3):L581-L591.

33. Heijink IH, Brandenburg SM, Postma DS, van Oosterhout AJ. Cigarette smoke impairs airway epithelial barrier function and cell-cell contact recovery. Eur Respir J. 2012;39(2):419-428.

34. Parmley LA, Elkins ND, Fini MA, Liu YE, Repine JE, Wright RM. Alpha-4/beta-1 and alpha-L/beta-2 integrins mediate cytokine induced lung leukocyte-epithelial adhesion and injury. Br J Pharmacol. 2007;152(6):915-929.

35. Michalet X, et al. Dynamic molecular combing: stretching the whole human genome for high-resolution studies. Science. 1997;277(5331):1518-1523.

36. Cho SH, Kim SK, Kwon E, Park HJ, Kwon KH, Chung JH. Polymorphism of IGF1R is associated with papillary thyroid carcinoma in a Korean population. J Interferon Cytokine Res. 2012;32(9):401-406.

37. Koike K, et al. Subcutaneous administration of neutralizing antibodies to endothelial monocyte-activating protein II attenuates cigarette smoke-induced lung injury in mice. Am J Physiol Lung Cell Mol Physiol. 2019;316(3):L558-L566.

38. Justice MJ, et al. Inhibition of acid sphingomyelinase disrupts LYNUS signaling and triggers autophagy. J Lipid Res 2018;59(4):596-606.

39. Subramanian A, et al. Gene set enrichment analysis: a knowledge-based approach for interpreting genome-wide expression profiles. Proc Natl Acad Sci USA. 2005;102(43):15545-15550.

40. Vaskovsky VE, Kostetsky EY, Vasendin IM. A universal reagent for phospholipid analysis. J Chromatogr. 1975;114(1):129-141.

41. Berdyshev EV, et al. De novo biosynthesis of dihydrosphingosine-1-phosphate by sphingosine kinase 1 in mammalian cells. Cell Signal. 2006;18(10):1779-1792. 